\title{
Evaluating Interest Rate Covariance Models within a
}

\author{
Value-at-Risk Framework*
}

\author{
Miguel A. Ferreira ${ }^{\dagger}$ \\ Jose A. Lopez ${ }^{\ddagger}$ \\ ISCTE School of Business \\ Federal Reserve Bank of San Francisco
}

This Version: March 2004

First Version: May 2003

\footnotetext{
* Please note that the views expressed here are those of the authors and not necessarily those of the Federal Reserve Bank of San Francisco or of the Federal Reserve System.

†Address: ISCTE Business School - Lisbon, Complexo INDEG/ISCTE, Av. Prof. Anibal Bettencourt, 1600-189 Lisboa, Portugal. Phone: 35121 7958607. Fax: 35121 7958605. E-mail: miguel.ferreira@iscte.pt.

${ }^{\ddagger}$ Address: Economic Research Department Group Risk Management, Federal Reserve Bank of San Francisco, 101 Market Street, San Francisco, CA 94705. Phone: (415) 977-3894. Fax: (415) 974-2168. E-mail: jose.a.lopez@sf.frb.org.
} 


\begin{abstract}
We find that covariance matrix forecasts for an international interest rate portfolio generated by a model that incorporates interest-rate level volatility effects perform best with respect to statistical loss functions. However, within a value-at-risk (VaR) framework, the relative performance of the covariance matrix forecasts depends greatly on the VaR distributional assumption. Simple forecasts based just on weighted averages of past observations perform best using a VaR framework. In fact, we find that portfolio variance forecasts that ignore the individual assets in the portfolio generate the lowest regulatory capital charge, a key economic decision variable for commercial banks. Our results provide empirical support for the commonly-used VaR models based on simple covariance matrix forecasts and distributional assumptions.
\end{abstract}

JEL classification: C52; C53; G12; E43

Keywords: Interest rates; Covariance models; GARCH; Forecasting; Risk Management, Value-atRisk 


\section{Introduction}

The short-term interest rate is one of the most important and fundamental variables in financial markets. In particular, interest rate volatility and its forecasts are important to a wide variety of economic agents. For example, portfolio managers require such volatility forecasts for asset allocation decisions; option traders require them to price and hedge interest rate derivatives; and risk managers use them to compute measures of portfolio market risk, such as value-at-risk (VaR). In fact, since the late 1990s, financial regulators have been using VaR methods and their underlying volatility forecasts to supervise and monitor the trading portfolios and interest rate exposures of financial institutions.

Forecasts of interest rate volatility are available from two general sources. The market-based approach uses option market data to infer implied volatility, which is interpreted to be the market's best estimate of future volatility conditional on an option pricing model. Since these forecasts incorporate the expectations of market participants, they are said to be forward-looking measures. The model-based approach forecasts volatility from time series data, generating what are usually called historical forecasts. The empirical evidence in Day and Lewis (1992), Amin and Ng (1997), and others suggests that implied volatility can forecast future volatility. However, there is also some consensus that implied volatility is an upward biased forecast of future volatility and that historical volatility forecasts contain additional information. Moreover, the scope of implied second moments is limited due to options contracts availability, especially with respect to covariances. ${ }^{1}$

Most of the work on the model-based approach to forecasting second moments has focused on univariate time-varying variance models. For examples, see Glosten, Jagannathan, and Runkle (1993) and Engle and Ng (1993). Most studies evaluate the performance of such volatility models in-sample analysis, although some have examined their out-of-sample performance using statistical loss functions; see for example, Pagan and Schwert (1990), West and Cho (1995), and Figlewski (1997).

Out-of-sample evaluation of forecasts provides an alternative and potentially more useful com-

\footnotetext{
${ }^{1}$ Campa and Chang (1998) and Walter and Lopez (2000) find that implied correlations from foreign exchange options tend to be more accurate forecasts of realized correlation than the ones based on historical prices. However, implied correlations do not fully incorporate the information in historical data because bivariate GARCH models of correlation are able to improve forecast accuracy.
} 
parison of the alternative models, due to the possibility of overfitting. ${ }^{2}$ Moreover, the ability to produce useful out-of-sample forecasts is the clear test of model performance because it only uses the same information set available to agents at each point in time. Previous studies across a wide variety of markets have compared the out-of-sample forecasting ability of several GARCH type models with that of simple variance measures and found that there is no clear winner using statistical loss functions. ${ }^{3}$ Results in Figlewski (1997) show that GARCH models fitted to daily data may be quite useful in forecasting the volatility in stock markets both for short and long horizons, but they are much less useful in making out-of-sample forecasts in other markets beyond short horizons $^{4}$. In particular, using 3-month US Treasury bill yields, sample variances generate more accurate forecasts than GARCH model. Ederington and Guan (1999) also find that sample variances dominate GARCH models in the 3-month Eurodollar market.

In contrast, relatively little work has been done on modeling and forecasting second moments using multivariate time-varying covariance models. Kroner and Ng (1998) propose a general family of multivariate GARCH models, called General Dynamic Covariance (GDC) model, to estimate the variance-covariance matrix of a portfolio of large and small US stocks. Ferreira (2001) presents a new multivariate model for interest rates, called the mixed GDC and levels (GDC-Levels) model, which includes both level effects and conditional heteroskedasticity effects in the volatility dynamics. ${ }^{5}$ He finds that the interest rate variance-covariance matrix is best forecasted out-of-sample using a VECH model with a level effect. In addition, simple models using either equally or exponentiallyweighted moving averages of past observations perform as well under statistical loss functions as the best multivariate model in terms of both variance and covariance. Overall, his out-of-sample results present evidence that model features often required to obtain a good in-sample fit do not contribute to out-of-sample forecasting power.

Given the wide variety of volatility models available and their mixed out-of-sample performance

\footnotetext{
${ }^{2}$ Lopez (2001) finds in the foreign exchange markets that out-of-sample analysis of volatility forecasts is useful because the best set of forecasts varies not only among foreign exchange rates but also from in-sample to out-of-sample evaluation. Ederington and Guan (1999) also find evidence supporting that in-sample results may not be confirmed out-of-sample.

${ }^{3}$ See Andersen and Bollerslev (1998) for further discussion of this point.

${ }^{4}$ This sensitivity of results to the forecast horizon can also be found in West and Cho (1995) with the GARCH model having a slight advantage for short forecast horizons in foreign exchange markets, but this advantage disappearing for longer horizons.

${ }^{5}$ The sensitivity of interest rate volatility to the interest rate level was first documented by Chan, Karolyi, Longstaff, and Sanders (1992).
} 
with respect to statistical loss functions, a key question is how to choose among them. A relatively informative method of model selection is to compare their out-of-sample performance in terms of a particular economic application and results may vary according to the selected economic loss function; see for example Lopez (2001). However, there has been relatively little work on comparing the out-of-sample performance of alternative models in terms of economic loss functions, especially in a multivariate framework.

To date, such economic evaluations of out-of-sample volatility forecasts has been in the areas of hedging performance, asset allocation decisions and options pricing. Regarding hedging strategies, Cecchetti, Cumby, and Figlewski (1988) find that a time-varying covariance matrix is necessary to construct an optimal hedge ratio between Treasury bonds and bond futures, while Kroner and Ng (1998) find that the choice of the multivariate specification can result in very different estimates of the optimal hedge ratio for stock portfolios. With respect to asset allocation, volatility model selection is also important as shown by Kroner and $\mathrm{Ng}$ (1998) in the context of a portfolio of small and large stocks. Poomimars, Cadle, and Theobald (2001) also find that for the purposes of allocating assets across national stock markets, simpler models of the covariance matrix are more appropriate when optimizing across asset universes that include less stable national markets and environments. For options pricing, Engle, Kane, and Noh (1997) and Gibson and Boyer (1998) find that standard time-series models produce better correlation forecasts than simple moving average models for the purpose of stock-index option pricing and trading. Christoffersen and Jacobs (2002) also evaluate alternative GARCH covariance forecasts in terms of option pricing and finds out-ofsample evidence in favor of a relatively parsimonious model.

In this paper, we consider economic loss functions related to financial risk management, which places emphasis on value-at-risk (VaR) measures that generally indicate the amount of portfolio value that could be lost over a given time horizon with a specified probability. Hendricks (1996) provides the most extensive evaluation of alternative VaR models using a portfolio of foreign exchange rates, although he does not examine covariance matrix forecasts. Other papers - Dave and Stahl (1996), Alexander and Leigh (1997), and Jackson, Maude, and Perraudin (1997) - examine VaR measures for different asset portfolios using multivariate models. We extend this literature by evaluating the forecast performance of multivariate volatility models within a VaR framework and using interest rate data, as per Lopez and Walter (2001) and Vlaar (2000), respectively. 
The performance of VaR for interest rate portfolios, one of the most important financial prices and of particular relevance in banks' trading portfolios ${ }^{6}$, has only been studied using univariate models. Vlaar (2000) examines the out-of-sample forecast accuracy of several univariate volatility models (equally and exponentially sample averages as well as GARCH models) for portfolios of Dutch bonds with different maturities. In addition, he examines the impact of alternative VaR distributional assumptions. The analysis shows that the percentage of exceedances for the simple models is basically equal to that for the GARCH models. The distributional assumption is important with the normal performing much better than the t-distribution.

In this paper, we analyze an equally-weighted portfolio of short-term fixed income positions in the US dollar, German deutschemark and Japanese yen. The portfolio returns are calculated from the perspective of a US-based investor using daily interest rate and foreign exchange data from 1979 to 2000. We examine VaR estimates for this portfolio from a wider variety of multivariate volatility models, ranging from simple sample averages to standard time-series models. We compare the out-of-sample forecasting performance of these alternative variance-covariance structures and their economic implications within the VaR framework used by Lopez and Walter (2001). The question of interest is whether more complex covariance models provide improved out-of-sample VaR forecasts.

The VaR framework we use consists of three sets of evaluation techniques. The first set focuses on the statistical properties of VaR estimates derived from alternative covariance matrix forecasts and VaR distributional assumptions. Specifically, the binomial test of correct unconditional and conditional coverage, which is implicitly incorporated into the aforementioned bank capital requirements, is used to examine $1 \%, 5 \%, 10 \%$ and $25 \%$ VaR estimates.

The second set of techniques focus on the magnitude of the losses experienced when VaR estimates are exceeded, clearly an issue of interest to financial institutions and their regulators. To determine whether the magnitudes of observed exceptions are in keeping with the model generating the VaR estimates, we use the hypothesis test based on the truncated normal distribution proposed by Berkowitz (2001).

Finally, to examine the performance of the competing covariance matrix forecasts with respect

\footnotetext{
${ }^{6}$ Berkowitz and O'Brien (2002) report that the poor performance of banks trading portfolios during the 1998-2000 period is mainly due to interest rate positions.
} 
to regulatory capital requirements, we use the regulatory loss function implied by the U.S. implementation of the market risk amendment to the Basel Capital Accord. This loss function penalizes a VaR model for poor performance by using a capital charge multiplier based on the number of VaR exceptions; see Lopez (1999b) for further discussion.

Our findings are roughly in line with those of Lopez and Walter (2001), which were based on a simple portfolio of foreign exchange positions. As per Ferreira (2001), we confirm that covariance matrix forecasts generated from models that incorporate interest-rate level effect perform best under statistical loss functions. However, within a VaR framework, we find that the relative performance of covariance matrix forecasts depends greatly on the VaR models' distributional assumptions. Of the VaR forecasts examined, simple specifications, such as exponentially-weighted moving averages of past observations, perform best with regard to the magnitude of VaR exceptions and regulatory capital requirements. Our results provide further empirical support for the commonly-used VaR models based on simple covariance matrix forecasts and distributional assumptions, such as the normal and the nonparametric distributions. Moreover, simple variance forecasts based only on the portfolio returns (i.e., ignoring the portfolio's component assets) perform as well as the best covariance forecasts of individual portfolio assets' and, in fact, generate the minimum capital requirements in our exercise.

These findings are also mainly consistent with the evidence in Berkowitz and O'Brien (2002), who analyzed the daily trading revenues and VaR estimates of six US commercial banks. They conclude that these VaR estimates are too high, but that losses do significantly exceed the VaR estimates at times within their sample period. They also find that a GARCH model of portfolio returns that ignores the component assets, when combined with a normal distributional assumption, provides VaR estimates and regulatory capital requirements that are comparable to other models and does not produce such large VaR exceptions. Our results based on fixed portfolio weights provide evidence that the Berkowitz and O'Brien (2002) finding is not explained by an offset between the static portfolio weights (i.e., current portfolio weights overestimate future portfolio weights if traders react to changes in volatility by adjusting their portfolio holdings in order to minimize risk) and the neglected leptokurtosis of the portfolio return distribution.

The paper is structured as follows. Section 2 describes the issue of forecasting variances and covariances and presents the alternative time-varying covariance specifications. We also compare 
the models in terms of out-of-sample forecasting power for variances and covariances. Section 3 describes the VaR models analyzed. Section 4 presents the results for the hypothesis tests that make up the VaR evaluation framework. Section 5 concludes.

\section{Time-Varying Covariance Models}

In this paper, we study an equally-weighted US dollar-denominated portfolio of three month US dollar (US\$), Deutschemark (DM) and Japanese yen (JY) interest rates. We take the view of a USbased investor, who is unhedged with respect to exchange rate risk. Thus, we need to estimate the (5x5) variance-covariance matrix between the three interest rates as well as DM/US\$ and JY/US\$ exchange rates.

\subsection{Forecasting Variances and Covariances}

The empirical model for the short-term interest rates $r_{i t}$ is given by, for $i=\mathrm{US} \$, \mathrm{DM}, \mathrm{JP}$,

$$
r_{i t}-r_{i t-1}=\mu_{i}+\kappa_{i} r_{i t-1}+\varepsilon_{i t}
$$

and for the foreign exchange rate $s_{i t}$, for $i=\mathrm{DM} / \mathrm{US} \$, \mathrm{JY} / \mathrm{US} \$$,

$$
\ln \left(\frac{s_{i t}}{s_{i t-1}}\right)=\varepsilon_{i t}
$$

with $E\left[\varepsilon_{i t} \mid \mathcal{F}_{t-1}\right]=0$ and where $\mathcal{F}_{t-1}$ is the information set up to time $t-1$. The conditional mean function for the interest rate in (1) is given by a first order autoregressive process and we assume a zero conditional mean for the continuously compounded foreign exchange rate of return in (2).

The conditional variance-covariance matrix $H_{t}$ is $(5 \times 5)$ with elements $\left[h_{i j t}\right]$. The conditional covariance can be expressed as

$$
h_{i j t}=E\left[\varepsilon_{i t} \varepsilon_{j t} \mid \mathcal{F}_{t-1}\right],
$$

and the conditional correlation is given by 


$$
\rho_{i j t}=\frac{E\left[\varepsilon_{i t} \varepsilon_{j t} \mid \mathcal{F}_{t-1}\right]}{\sqrt{E\left[\varepsilon_{i t} \mid \mathcal{F}_{t-1}\right] E\left[\varepsilon_{j t} \mid \mathcal{F}_{t-1}\right]}}=\frac{h_{i j t}}{\sqrt{h_{i i t} h_{j j t}}} .
$$

The first category of forecasts is based on sample variances-covariances using past data. Many sample estimators have been proposed for the variance and covariance. The most popular is the equally-weighted sample covariance, over the $R$ week historical period,

$$
\widehat{h}_{i j t}=\frac{1}{R} \sum_{s=0}^{R-1} \varepsilon_{i t-s} \varepsilon_{j t-s} .
$$

where $\varepsilon_{i t-s}$ is the return $i$ on week $t-s$. The equally-weighted sample variance is a special case of the previous expression when $i=j$. This forecast requires the selection of the amount of data in computing the sample covariance. This is usually done using a fixed amount of data (up to the estimation time $t$ ) and the resulting forecast is named of rolling sample covariance forecast. The sample covariance estimator weights equally each past observation included in the estimate. We consider two alternatives sample averages: the last 250 days (E250) ${ }^{7}$ and all past observations (Eall). However, a common feature with financial data is that recent observations are more meaningful than older ones. A way to incorporate this feature is to weight observations in inverse relation to their age.

The exponentially-weighted sample covariance (EWMA) assigns more weight to recent observations relative to more distant observations. Let $w$ be the weight factor (decay rate) with $0<w \leq 1$. The forecast formulated at time $t$ is obtained using all available observations up to the estimation time $t$,

$$
\widehat{h}_{i j t}=\frac{\sum_{s=0}^{t-1} w^{s} \varepsilon_{i t-s} \varepsilon_{j t-s}}{\sum_{s=0}^{t-1} w^{s}} .
$$

The exponentially-weighted sample variance is a special case of the previous expression ${ }^{8}$. In the limit, the forecasts given by equation (6) include an infinite number of past observations, but with those from a more distant past having assigned infinitesimally small weights. The magnitude of the weight factor determines the degree to which more recent data is weighted. This is equivalent to the

\footnotetext{
${ }^{7}$ This window corresponds to the minimum amount of data allowed to formulate VaR forecasts for regulatory capital purposes.

${ }^{8}$ The exponentially-weighted sample variance forecast is equivalent to a $\operatorname{GARCH}(1,1) \operatorname{model}$ with $\beta_{0}=0, \beta_{1}=w$ and $\beta_{2}=1-w$, i.e. corresponds to an IGARCH model with zero constant.
} 
exponential smoother proposed in the Riskmetrics. The exponentially-weighted forecast requires the estimation of the weight factor $w$. We assume a weight factor $w=0.94$, which it is identical to the one commonly used in practice (e.g. Riskmetrics).

We also consider forecasts based directly on the portfolio returns, which ignore the covariance matrix specification completely. This approach significantly reduces the computational time (and estimation errors) and presents comparable performance in terms of VaR as reported in Lopez and Walter (2001) and Berkowitz and O'Brien (2002). In particular, we examine the equallyweighted (with 250 and all past observations) and exponentially-weighted (with a decay factor of 0.94) sample variance of portfolio returns and denote them of E250 Portfolio, Eall Portfolio, and EWMA Portfolio, respectively. We also consider a $\operatorname{GARCH}(1,1)$ variance model of portfolio return, denoted GARCH Portfolio.

Multivariate GARCH models are a natural extension of the previous methods and are among the most widely used time-varying covariance models. In the basic multivariate GARCH model, the components of the conditional variance-covariance matrix $H_{t}$ vary through time as functions of the lagged values of $H_{t}$ and of the squared innovations, $\varepsilon_{t}$ is $(5 \times 1)$ vector of innovations. We model the US\$, DM and JP short-term interest rates as well as DM/US\$ and JY/US\$ foreign exchange rates using a multivariate $\operatorname{GARCH}(1,1)$ structure because this process is able to successfully represent most financial time series. We implement several (but not all) multivariate GARCH specifications that have been suggested in the literature as we will discuss below. ${ }^{9}$

\subsubsection{VECH Model}

The VECH model of Bollerslev, Engle, and Wooldridge (1988) is a parsimonious version of the most general multivariate GARCH model, and is given by, for $i, j=1,2, \ldots, 5$,

$$
h_{i j t}=\omega_{i j}+\beta_{i j} h_{i j t-1}+\alpha_{i j} \varepsilon_{i t-1} \varepsilon_{j t-1}
$$

where $\omega_{i j}, \beta_{i j}$ and $\alpha_{i j}$ are parameters. The VECH model is simply an ARMA process for $\varepsilon_{i t} \varepsilon_{j t}$. The covariance is estimated using geometrically declining weighted averages of past cross products of innovations to the interest rate process. An implementation problem is that the model may not

\footnotetext{
${ }^{9}$ A notable exception is the recently proposed DCC model by Engle (2002).
} 
yield a positive definite covariance matrix. The VECH model does not allow for volatility spillover effects, i.e. the conditional variance of a given variable is not a function of other variable shocks and past variance.

\subsubsection{Constant Correlation (CCORR) Model}

In the CCORR model of Bollerslev (1990), the conditional covariance is proportional to the product of the conditional standard deviations. Consequently, the conditional correlation is constant across time. The model is described by the following equations, for $i, j=1,2, \ldots, 5$,

$$
h_{i i t}=\omega_{i i}+\beta_{i i} h_{i i t-1}+\alpha_{i i} \varepsilon_{i t-1}^{2}, \quad h_{i j t}=\rho_{i j} \sqrt{h_{i i t-1}} \sqrt{h_{j j t-1}}, \quad \text { for all } i \neq j .
$$

The conditional covariance matrix of the CCORR model is positive definite if and only if the correlation matrix is positive definite. Like in the VECH model there is no volatility spillover effects across series.

\subsubsection{BEKK Model}

The BEKK model of Engle and Kroner (1995) addresses the problem of the estimated conditional covariance matrix being positive definite. The model is given by:

$$
H_{t}=\Omega+B^{\prime} H_{t-1} B+A^{\prime} \varepsilon_{t-1} \varepsilon_{t-1}^{\prime} A,
$$

where $\Omega, A$, and $B$ are $(5 \times 5)$ matrices, with $\Omega$ symmetric. In the BEKK model, the conditional covariance matrix is determined by the outer product matrices of the vector of past innovations. As long as $\Omega$ is positive definite, the conditional covariance matrix is also positive definite because the other terms in (9) are expressed in quadratic form.

\subsubsection{General Dynamic Covariance (GDC) Model}

The GDC model proposed by Kroner and Ng (1998) nests many of the existing multivariate GARCH models. The conditional covariance specification is given by:

$$
H_{t}=D_{t} R D_{t}+\Phi \circ \Theta_{t}
$$


where $\circ$ is the Hadamard product operator (element-by-element matrix multiplication), $D_{t}$ is a diagonal matrix with elements $\sqrt{\theta_{i i t}}, R=\left[\rho_{i j}\right]$ is the correlation matrix, $\Phi=\left[\phi_{i j}\right]$ is a matrix of parameters with $\phi_{i i}=0$ for all $i, \Theta_{t}=\left[\theta_{i j t}\right]$,

$$
\theta_{i j t}=\omega_{i j}+b_{i}^{\prime} H_{t-1} b_{j}+a_{i}^{\prime} \varepsilon_{t-1} \varepsilon_{t-1}^{\prime} a_{j}, \quad \text { for all } i, j
$$

$a_{i}, b_{i}, i=1,2, \ldots, 5$ are $(5 \times 1)$ vectors of parameters, and $\omega_{i j}$ are scalars with $\Omega \equiv\left[\omega_{i j}\right]$ being a diagonal positive definite $(5 \times 5)$ matrix.

This model combines the constant correlation model with the BEKK model. The first term in (10) is like the constant correlation model but with variance functions given by the BEKK model. The second term in (10) has zero diagonal elements with the off-diagonal elements given by the BEKK model covariance functions scaled by the $\phi_{i j}$ parameters. Thus, the GDC model structure can be written as follows:

$$
\begin{aligned}
& h_{i i t}=\theta_{i i t} \\
& h_{i j t}=\rho_{i j} \sqrt{\theta_{i i t}} \sqrt{\theta_{j j t}}+\phi_{i j} \theta_{i j t} \quad \text { for all } i \neq j .
\end{aligned}
$$

The GDC model nests the other multivariate GARCH models discussed above ${ }^{10}$. The VECH model assumes that $\rho_{i j}=0$ for all $i \neq j .{ }^{11}$ The BEKK model has the restrictions $\rho_{i j}=0$ for all $i \neq j$ and $\phi_{i j}=1$ for all $i \neq j$. The CCORR model considers $\phi_{i j}=0$ for all $i \neq j$.

\subsubsection{GDC-Levels (GDC-L) Model}

One of the most important features of the short-term interest rate volatility is the level effect, i.e. volatility is a function of the interest rate level. Chan et al. (1992) was the first paper to document this effect considering a conditional variance specification where the interest rate level solely drive the evolution of the interest rate volatility. They found a volatility sensitivity to the interest rate level very high in excess of the unity. However, subsequent work by Brenner, Harjes, and Kroner

\footnotetext{
${ }^{10}$ See proposition 1 in Kroner and $\mathrm{Ng}(1998)$.

${ }^{11}$ The GDC model does not nest exactly the VECH model but a restricted version of it with restrictions $\beta_{i j}=\beta_{i i} \beta_{j j}$ and $\alpha_{i j}=\alpha_{i i} \alpha_{j j}$.
} 
(1996) show that the level effect exists but it is considerably smaller (consistent with a square root process) by incorporating in the variance function both level and conditionally heteroskedasticity effect (news effect) in a GARCH framework. Bali (2003) also finds that incorporating both GARCH and level effects in the interest rate volatility specifications improves the pricing performance of the models. Here, we consider a multivariate version of the Brenner et al. (1996) model combining a multivariate GARCH model with the level effec.

The GDC model of Kroner and Ng (1998) can be extended to incorporate level effect in the variance-covariance function for the interest rates (not for the exchange rates),

$$
\theta_{i j t}=\omega_{i j}^{*}+b_{i}^{\prime} H_{t-1}^{*} b_{j}+a_{i}^{\prime} \varepsilon_{t-1}^{*} \varepsilon_{t-1}^{* \prime} a_{j}, \quad \text { for } i, j=1,2,3
$$

where $\omega_{i i}^{*}=\omega_{i i} / r_{i t-1}^{2 \gamma i}$, the diagonal elements of the matrix $H_{t-1}^{*}$ are multiplied by the factor $\left(r_{i t-1} / r_{i t-2}\right)^{2 \gamma i}$ for all $i$, and the elements of the vector $\varepsilon_{t-1}^{*}$ are divided by $r_{i t-1}^{\gamma i}$ for all $i$. The GDCLevels model nests the other multivariate GARCH models combined with level effect. The exact form of conditional variance in the VECH model with level effect (VECH-Levels model, VECH-L) for the interest rates is given by:

$$
h_{i i t}=\left(\omega_{i i}+\beta_{i i} h_{i i t-1} r_{i t-2}^{-2 \gamma_{i}}+\alpha_{i i} \varepsilon_{i t-1}^{2}\right) r_{i t-1}^{2 \gamma_{i}} .
$$

The CCORR model with level effect (CCORR-Levels, CCORR-L) will also have a variance function given by the equation (14) and the covariance function is defined as before. Similarly, we also incorporate level effect within the BEKK specification (BEKK-Levels model, BEKK-L).

\subsection{Models Estimation}

\subsubsection{Data Description}

The interest rate data consists of US dollar (US\$), Deutschemark (DM) and Japanese yen (JY) London interbank money market three-month interest rates (middle rate) obtained from Datastream (codes ECUSD3M, ECWGM3M and ECJAP3M, respectively). ${ }^{12}$ The interest rates are given in percentage and in annualized form. These interest rates are taken as proxies for the instantaneous

\footnotetext{
${ }^{12}$ The only exception is the JY interest rate in the period from January 1995 to December 2000 in which we use the three-month offered rate (code JPIBK3M).
} 
riskless interest rate (i.e., the short rate). We also require foreign exchange rates to compute U.S. dollar investor rates of return. The exchange rate market data includes JY per US\$ (denoted JY/US\$) and DM per US\$ (denoted DM/US\$) spot exchange rates in the London market also from Datastream. ${ }^{13}$

The frequency is daily and covers the period from January 12, 1979 to December 29, 2000, providing 5731 observations in total. Table 1 presents summary statistics for the interest and exchange rates. The daily mean interest rate changes are close to zero. The daily interest rate changes standard deviation differ considerably across countries with the US dollar interest rate having an annualized standard deviation of $2.8 \%$ compared with $1.8 \%$ and $1.4 \%$ for the Japanese and German interest rates, respectively. Foreign exchange rates present a much higher degree of variation with an annualized standard deviation of about $11 \%$ for both series. Table 1 also presents summary statistics for an equally-weighted portfolio of the US\$, DM and JY interest rates with returns calculated in US\$ (see section 3 for a detailed portfolio definition). The mean portfolio return is close to zero with an annualized standard deviation of $19.6 \%$. The portfolio return presents leptokurtosis, though less severe that most individual components series, with exception of the DM/US\$ exchange rate return.

As in most of the literature, we focus on nominal interest rates because defining real interest rates, especially at high frequencies, is subject to serious measurement errors. The use of daily frequency minimizes the discretization bias resulting from estimating a continuous-time process using discrete periods between observations. On the other hand, the use of a three-month rate maturity proxy for the instantaneous interest rate is unlikely to create a significant proxy bias as shown in Chapman, Long, and Pearson (1999). Finally, we use interbank interest rates instead of Treasury bill yields because of the low liquidity of the Treasury bill secondary market in both Germany and Japan, in sharp contrast with the high liquidity of the interbank money market. The concern regarding the existence of a default premium in the interbank money market should not be severe since these interest rates result from trades among high-grade banks that are considered almost default-free.

\footnotetext{
${ }^{13}$ For earlier years of the sample, the direct per US\$ quotes are not available, but we construct the series from British pound (BP) exchange rates quoted by WM/Reuters (US\$ per BP, DM per BP and JY per BP rates).
} 


\subsubsection{Forecasting Performance}

We estimate the multivariate models parameters using the period from January 12, 1977 to December 29, 1989 (2861 daily observations) to calculate the models' one-step-ahead variance-covariance forecasts over the out-of-sample period from January 1, 1990 and December 29, 2000 (2870 observations). We use a fixed scheme to estimate the multivariate models' parameters, i.e. we do not reestimate the parameters as we move forward in the out-of-sample period.

Following standard practices, we estimate the models by maximizing the likelihood function under the assumption of independent and identically distributed (i.i.d.) innovations. In addition, we assume that the conditional density function of the innovations is given by the normal distribution. Conditional normality of the innovations is often difficult to justify in many empirical applications that use leptokurtic financial data. However, the maximum likelihood estimator based on the normal density has a valid quasi-maximum likelihood (QML) interpretation as shown by Bollerslev and Wooldridge (1992). ${ }^{14}$

Tables 2 and 3 presents models' out-of-sample forecasting performance in terms of root mean squared prediction error (RMPSE). Table 2 reports RMSPE for the variances of the five component assets and the overall portfolio, and Table 3 reports RMSPE for ten relevant covariances. We also report the Diebold and Mariano (1995) test statistic comparing the models' forecasting accuracy relative to the one with the minimum RMSPE.

Overall, the best performing interest rate volatility models are the VECH and CCORR models with a level effect; note that including volatility spillover effects is not important. The null hypothesis that the VECH-Levels and CCORR-Levels models perform similarly to the best forecast is only rejected at the $5 \%$ level in 3 and 2 cases out of 15 cases (i.e., five variances and ten covariances), respectively. The VECH-Levels and CCORR-Levels model are among the two best performing models in 6 and 4 cases, respectively.

A simple model using equally-weighted moving averages of past 250 observations (E250) performs similarly to the best multivariate models, especially in terms of covariance. Also, the exponentially-weighted moving average (EWMA) forecast also performs quite well for variances, though for covariances presents low accuracy in some cases. The null hypothesis of equality between

\footnotetext{
${ }^{14}$ Model parameters are not presented here in order to conserve space, but they are available from the authors upon request.
} 
this forecast and the minimizing forecast is only rejected at the $5 \%$ level in 3 cases and it is one of the two RMSPE minimizing forecasts in 8 cases out of 15 cases.

The worst performing models are the GDC and GDC-Levels models, which are the most general specifications and indicate that they tend to overfit the data in-sample. The GDC-Levels model is rejected in 9 cases at the $5 \%$ level and in 3 cases (out of 15 ) at the $10 \%$ level in terms of generating forecasts with similar performance to the best model. Among the simple models, the equally-weighted moving average of all past observations (Eall) presents the worst performance being rejected at the $5 \%$ level in 13 cases out of 15 cases.

The model selection is more important for forecasting interest rates variance, especially for the US, than for forecasting exchange rates volatility. Also, the performance of covariance forecasts are more sensitive to model selection than the performance of variance forecasts.

With respect to the portfolio variance, it is best forecast by the VECH-Levels model confirming the models forecasting performance in terms of individual variances and covariances. The null hypothesis of equal forecasting power relative to the best model is not rejected for the portfolio variance forecasts that are based directly on the portfolio returns (GARCH Portfolio and EWMA Portfolio). This result indicates that simple portfolio variance forecasts are useful, but that forecasts based on covariance matrix specifications generally perform as well or better. Surprisingly, the equally-weighted moving averages of past 250 observations forecast (E250) is rejected in terms of forecasting portfolio variance, in contrast with its good performance for individual variances and covariances.

In summary, statistical loss functions provide a useful, although incomplete, analysis of individual assets' variance and covariance as well as portfolio variance forecasts. Our results indicate that the second moment forecasts from simple models and time-varying covariance models present similar out-of-sample forecasting performance. In addition, the most complex time-varying covariance models tend to overfit the data. Our findings are generally consistent with those of Ferreira (2001) for interest rates, and West and Cho (1995) and Lopez (2001) for exchange rates. However, economic loss functions that explicitly incorporate the costs faced by volatility forecast users provide the most meaningful forecast evaluations. In the next section, we examine the performance of the covariance matrix forecasts within a value-at-risk framework. 


\section{Value-at-Risk Framework}

\subsection{Portfolio Definition}

Assume that we have a US fixed-income investor managing positions in US\$, DM, and JY zerocoupon bonds ${ }^{15}$ in terms of dollar-denominated returns ${ }^{16}$. The value of a zero-coupon bond with unit face value and a three-month maturity at time $t+1$ is given by

$$
P_{\mathrm{US} \$ t+1}=e^{-\frac{1}{4} r_{\mathrm{US} \$ t+1}},
$$

where $r_{\mathrm{US} \$ t+1}$ is the continuously compounded three-month interest rate (annualized). Define the value of a US\$ investment tomorrow to be

$$
Y_{\mathrm{US} \$ t+1}=Y_{\mathrm{US} \$ t} e^{R_{\mathrm{US} \$ t+1}},
$$

where $R_{\mathrm{US} \$ t+1}$ denotes the day $t+1$ geometric rate of return on the three-month zero-coupon bond investment. In the US case, $Y_{\mathrm{US} \$ t+1}=P_{\mathrm{US} \$ t+1}$, hence

$$
R_{\mathrm{US} \$ t+1}=-\frac{1}{4}\left(r_{\mathrm{US} \$ t+1}-r_{\mathrm{US} \$ t}\right) .
$$

The value of the DM zero-coupon bond in US\$ tomorrow is given by:

$$
Y_{\mathrm{DM} t+1}=\left(Y_{\mathrm{DM} t} s_{\mathrm{DM} / \mathrm{US} \$ t}\right) e^{R_{\mathrm{DM} i t+1}} s_{\mathrm{US} \$ / \mathrm{DM} t+1},
$$

where $s_{\mathrm{US} \$ / \mathrm{DM} t}$ is the exchange rate between US\$ and DM. Thus, the day $t+1$ geometric rate of return on the DM three-month zero-coupon bond investment given by:

$$
\ln \left(Y_{\mathrm{DM} t+1}\right)-\ln \left(Y_{\mathrm{DM} t}\right)=R_{\mathrm{DM} t+1}-\Delta \ln \left(s_{\mathrm{DM} / \mathrm{US} \$ t+1}\right),
$$

\footnotetext{
${ }^{15}$ We use bond returns implied by interbank money market rates (LIBOR) instead of bond returns. This is common to other studies such as Duffie and Singleton (1997) and presents several advantages. First, the interbank money market is more liquid than the Treasury bill market in most countries. Second, most interest rate derivatives are priced using interbank interest rates. Third, credit risk minimally affects these rates as they are subject to special contractual netting features and correspond to trades among high-grade banks.

${ }^{16}$ We take the perspective of a US-based investor, who is unhedged with respect to exchange rate risk.
} 
where $R_{\mathrm{DM} t+1}=-\frac{1}{4}\left(r_{\mathrm{DM} t+1}-r_{\mathrm{DM} t}\right)$ is the local-currency rate of return on the fixed income investment and $\Delta \ln \left(s_{\mathrm{DM} / \mathrm{US} \$ t+1}\right)=\left[\ln \left(s_{\mathrm{DM} / \mathrm{US} \$ t+1}\right)-\ln \left(s_{\mathrm{DM} / \mathrm{US} \$ t}\right)\right]$ is the foreign exchange rate of return. The analysis for the JY fixed income position is the same as for the DM fixed income, with the geometric rate of return on the JY three-month zero-coupon bond investment given by

$$
\ln \left(Y_{\mathrm{JY} t+1}\right)-\ln \left(Y_{\mathrm{JY} t}\right)=R_{\mathrm{JY} t+1}-\Delta \ln \left(s_{\mathrm{JY} / \mathrm{US} \$ t+1}\right)
$$

where $R_{\mathrm{JY} t+1}=-\frac{1}{4}\left(r_{\mathrm{JY} t+1}-r_{\mathrm{JY} t}\right)$ and $\Delta \ln \left(s_{\mathrm{JY} / \mathrm{US} \$ t+1}\right)=\left[\ln \left(s_{\mathrm{JY} / \mathrm{US} \$ t+1}\right)-\ln \left(s_{\mathrm{JY} / \mathrm{US} \$ t}\right)\right]$.

For simplicity, we will assume that the portfolio at time $t$ is evenly divided between the three fixed-income positions (equally-weighted portfolio). Thus, the investor's market risk exposure is to the sum of the three investment returns. That is,

$$
R_{p t+1}=\left[\begin{array}{lllll}
R_{\mathrm{US} \$ t+1} & R_{\mathrm{DM} t+1} & \Delta \ln \left(s_{\mathrm{DM} / \mathrm{US} \$ t+1}\right) & R_{\mathrm{JY} t+1} & \Delta \ln \left(s_{\mathrm{JY} / \mathrm{US} \$ t+1}\right)
\end{array}\right] w^{\prime},
$$

where $w=\left[\begin{array}{lllll}1 & 1 & -1 & 1 & -1\end{array}\right]$ is the portfolio weight vector.

\subsection{VaR Models and Exceptions}

We examine the accuracy of several VaR models based on different covariance matrix specifications and conditional densities. A VaR estimate on day $t$ is a specified quantile of the forecasted return distribution $f_{t+k}$ over a given holding period $k$. The VaR estimate at time $t$ derived from model $m$ for a $k$-period-ahead return, denoted $\operatorname{VaR}_{m t}(k, \alpha)$, is the critical value that corresponds to the lower $\alpha$ percent tail of the distribution,

$$
\operatorname{VaR}_{m t}(k, \alpha)=F_{m t+k}^{-1}(\alpha)
$$

where $F_{m t+k}^{-1}$ is the inverse of the cumulative distribution function to $f_{m t+k}$. A VaR model $m$ consists of two items: a covariance matrix model and a univariate distributional assumption. Following Lopez and Walter (2001), we separately specify the portfolio variance dynamics

$$
h_{m p t+1}=w^{\prime} H_{m t+1} w,
$$


and the distribution form of $f_{m t+k} \cdot{ }^{17}$ In terms of portfolio variance dynamics we consider eight multivariate covariance model forecasts, three sample variance forecast and four portfolio variance forecasts, in a total of 15 . In terms of distributions we consider four alternative assumptions: normal, t-student, generalized t-student, and nonparametric distribution. This gives a total of $60 \mathrm{VaR}$ models. The last distributional form is the unsmoothed distribution of the standardized portfolio returns residuals, which is the distribution used in the so-called "historical simulation" approach for generating VaR estimates.

The t-distribution assumption requires an estimate of the number of degrees of freedom, which affects the tail thickness of the distribution. This parameter is estimated using the in-sample standardized residuals generated by the 15 model specifications. The estimated number of degrees of freedom ranges from 8 and 11, which correspond to some fat taildness, and the implied $1 \%$ quantiles range from -2.9 to -2.7 compared with -2.33 for the normal distribution. The generalized t-distribution assumption requires estimates of two parameters that control the tail thickness and width of the center of the distribution, named $n$ and $q$. The parameters $n$ and $q$ are also estimated using the in-sample standardized residuals and are found to be approximately to 2.5 and 1, respectively. These estimates imply considerable more fat taildness than the normal distribution at the $1 \%$ quantile (-3.27), but the reverse is true at the higher quantiles.

The nonparametric distribution quantiles are estimated using the unsmoothed distribution of the standardized portfolio return residuals arising from each of the 15 models. The estimated nonparametric critical values generally present more fat-taildness at the $1 \%$ and $5 \%$ quantiles than the normal distribution, but the opposite is true at the higher quantiles.

The one-day-ahead VaR estimates from model $m$ for the portfolio at the $\alpha$ quantile on day $t$ is

$$
\operatorname{VaR}_{m t}(\alpha)=\sqrt{h_{m p t+1}} F_{m}^{-1}(\alpha)
$$

where we drop the subscript $t$ from the portfolio distribution because we assume that it is constant across time. We then compare $\operatorname{VaR}_{m t}(\alpha)$ to the actual portfolio return on day $t+1$, denoted

\footnotetext{
${ }^{17}$ The intuition for specifying a VaR model as a separate portfolio variance (whether based on a modeled covariance matrix or portfolio variance) and a distributional assumption arises from the two-step procedure proposed by Engle and Gonzalez-Rivera (1991). Note that an alternative approach is to estimate the parameters of a multivariate volatility model using a distributional form other than the multivariate normal. However, such distributional forms are difficult to specify and use in estimation.
} 
as $R_{p t+1}$. If $R_{p t+1}<\operatorname{VaR}_{m t}(\alpha)$, then we have an exception. For testing purposes, we define the exception indicator variable as

$$
I_{m t+1}=\left\{\begin{array}{cl}
1 & \text { if } R_{p t+1}<\operatorname{VaR}_{m t}(\alpha) \\
0 & \text { if } R_{p t+1} \geq \operatorname{VaR}_{m t}(\alpha)
\end{array} .\right.
$$

\subsection{VaR Evaluation Tests}

\subsubsection{Unconditional and Conditional Coverage Tests}

Assuming that a set of VaR estimates and their underlying model are accurate, exceptions occur when $R_{p t+1}<\operatorname{VaR}_{m t}(\alpha)$, and they can be modeled as independent draws from a binomial distribution with a probability of occurrence equal to $\alpha$ percent. Accurate VaR estimates should exhibit the property that their unconditional coverage $\widehat{\alpha}=x / T$ equals $\alpha$ percent, where $x$ is the number of exceptions and $T$ the number of observations. The likelihood ratio statistic for testing whether $\widehat{\alpha}=\alpha$ is

$$
L R_{u c}=2\left[\log \left(\widehat{\alpha}^{x}(1-\widehat{\alpha})^{T-x}\right)-\log \left(\alpha^{x}(1-\alpha)^{T-x}\right)\right]
$$

which has an asymptotic $\chi^{2}(1)$ distribution. ${ }^{18}$

The $L R_{u c}$ test is an unconditional test of the coverage of VaR estimates since it simply counts exceptions over the entire period without reference to the information available at each point in time. However, if the underlying portfolio returns exhibit time-dependent heteroskedasticity, the conditional accuracy of VaR estimates is probably a more important issue. In such cases, VaR models that ignore such variance dynamics will generate VaR estimates that may have correct unconditional coverage, but at any given time, will have incorrect conditional coverage.

To address this issue, Christoffersen (1998) proposed conditional tests of VaR estimates based on interval forecasts. VaR estimates are essentially interval forecasts of the lower $1 \%$ tail of $f_{m t+1}$,

\footnotetext{
${ }^{18}$ The finite sample distribution of the $L R_{u c}$ statistic as well as the others in this study are of interest in actual practice; see Lopez (1999a) and Lopez (1999b). For example, with respect to size, the finite sample distribution of $L R_{u c}$ for specified $(\alpha, T)$ values may be sufficiently different from a $\chi^{2}(1)$ distribution that the asymptotic critical values may be inappropriate. As for the power of this test, Kupiec (1995) shows how this test has a limited ability to distinguish among alternative hypotheses and thus has low power in the typical samples of size 250 used for risk management purposes. However, since 2,870 out-of-sample observations are used in this exercise, the asymptotic distributions for all of the test statistics are used.
} 
the one-step-ahead return distribution. The $L R_{c c}$ test used here is a test of correct conditional coverage. Since accurate VaR estimates have correct conditional coverage, the $I_{m t+1}$ variable must exhibit both correct unconditional coverage and serial independence. The $L R_{c c}$ test is a joint test of these properties, and the relevant test statistic is $L R_{c c}=L R_{u c}+L R_{i n d}$, which is asymptotically distributed $\chi^{2}(2)$.

The $L R_{\text {ind }}$ statistic is the likelihood ratio statistic for the null hypothesis of serial independence against the alternative of first-order Markov dependence. ${ }^{19}$ The likelihood function under this alternative hypothesis is

$$
L_{A}=\left(1-\pi_{01}\right)^{T_{00}} \pi_{01}^{T_{01}}\left(1-\pi_{11}\right)^{T_{10}} \pi_{11}^{T_{11}}
$$

where the $T_{i j}$ notation denotes the number of observations in state $j$ after having been in state $i$ in the previous period, $\pi_{01}=T_{01} /\left(T_{00}+T_{01}\right)$ and $\pi_{11}=T_{11} /\left(T_{10}+T_{11}\right)$. Under the null hypothesis of independence, $\pi_{01}=\pi_{11}=\pi$, and the likelihood function is

$$
L_{0}=(1-\pi)^{T_{00}+T_{01}} \pi^{T_{01}+T_{11}}
$$

where $\pi=\left(T_{01}+T_{11}\right) / T$. The test statistic

$$
L R_{\text {ind }}=2\left[\log L_{A}-\log L_{0}\right]
$$

has an asymptotic $\chi^{2}(1)$ distribution.

\subsubsection{Dynamic Quantile Test}

We also use the Dynamic Quantile $(D Q)$ test proposed by Engle and Manganelli (1999), which is a Wald test of the hypothesis that all coefficients as well as the intercept are zero in a regression of the exception indicator variable on its past values (we use 5 lags) and on current VaR, i.e.

$$
I_{m t+1}=\delta_{0}+\sum_{k=1}^{5} \delta_{k} I_{m t-k+1}+\delta_{6} V a R_{m t}+\epsilon_{t+1}
$$

\footnotetext{
${ }^{19}$ As discussed in Christoffersen (1998), several other forms of dependence, such as second-order Markov dependence, can be specified. For the purposes of this paper, only first-order Markov dependence is used.
} 
We estimate the regression model by OLS, and a good model should produce a sequence of unbiased and uncorrelated $I_{m t+1}$ variable, so that the explanatory power of this regression should be zero.

\subsubsection{Distribution Forecast Test}

Since VaR models are generally characterized by their distribution forecasts of portfolio returns, several authors have suggested that evaluations should be based directly on these forecasts. Such an evaluation would use all of the information available in the forecasts. The object of interest in these evaluation methods is the observed quantile $q_{m t+1}$, which is the quantile under the distribution forecast $f_{m t+1}$ in which the observed portfolio return $R_{p t+1}$ actually falls; i.e.,

$$
q_{m t+1}\left(R_{p t+1}\right)=\int_{-\infty}^{R_{p t+1}} f_{m t+1}\left(R_{p}\right) d R_{p}
$$

If the underlying VaR model is accurate, then its $q_{m t+1}$ series should be independent and uniformly distributed over the unit interval.

Several hypothesis tests have been proposed for testing these two properties. Here, we use the likelihood ratio test proposed by Berkowitz $(2001)^{20}$. To examine whether the $q_{m t+1}$ series exhibits these properties, the $z_{m t+1}$ series is generated by transforming the $q_{m t+1}$ series with the inverse of the standard normal cumulative distribution function; i.e. $z_{m t+1}=\Phi^{-1}\left(q_{m t+1}\right)$. If the VaR model is correctly specified, the $z_{m t+1}$ series should be independent and identically distributed as standard normals. This hypothesis can be tested against alternative specifications, such as

$$
z_{m t+1}-\mu_{m}=\rho_{m}\left(z_{m t}-\mu_{m}\right)+\eta_{t+1},
$$

where the parameters $\left(\mu_{m}, \rho_{m}\right)$ are respectively the conditional mean and $\operatorname{AR}(1)$ coefficient corresponding to the $z_{m t+1}$ series and $\eta_{t+1}$ is a normal random variable with mean zero and variance $\sigma_{m}^{2}$. Under the null hypothesis that both properties are present, $\left(\mu_{m}, \rho_{m}, \sigma_{m}^{2}\right)=(0,0,1)$. The appropriate $L R$ statistic is

$$
L R_{\text {dist }}=2\left[L\left(\mu_{m}, \rho_{m}, \sigma_{m}^{2}\right)-L(0,0,1)\right],
$$

\footnotetext{
${ }^{20}$ Crnkovic and Drachman (1996) suggest that these two properties be examined separately and thus propose two separate hypothesis tests. Diebold, Gunther, and Tay (1998) propose the use of CUSUM statistic to test for these properties simultaneously. Christoffersen and Pelletier (2003) propose duration-based tests.
} 
where $L\left(\mu_{m}, \rho_{m}, \sigma_{m}^{2}\right)=-\frac{1}{2} \log \left(\frac{2 \pi \sigma_{m}^{2}}{1-\rho_{m}^{2}}\right)-\frac{\left(z_{m t+1}-\frac{\mu_{m}}{1-\rho_{m}}\right)^{2}}{2 \sigma_{m}^{2} /\left(1-\rho_{m}^{2}\right)}-\frac{(T-1) \log \left(2 \pi \sigma_{m}^{2}\right)}{2}-\sum_{t=1}^{T-1} \frac{\left(z_{m t+1}-\mu_{m}-\rho_{m} z_{m t}\right)^{2}}{2 \sigma_{m}^{2}}$. The $L R_{\text {dist }}$ statistic is asymptotically distributed $\chi^{2}(3)$.

\subsubsection{Exception Magnitudes}

The evaluation of VaR models, both in practice and in the literature, has generally focused on the frequency of exceptions and thus has disregarded information on their magnitudes. However, as discussed by Hendricks (1996) and Berkowitz (2001), the magnitudes of exceptions should be of primary interest to the various users of VaR models.

We use an evaluation method that focuses on the magnitude of the losses experienced when VaR estimates are exceeded. Berkowitz (2001) proposes a hypothesis test for determining whether the magnitude of observed VaR exceptions are consistent with the underlying VaR model. The key intuition is that VaR exceptions are treated as continuous random variables and not converted into the binary $I_{m t+1}$ variable used for the binomial tests. For this test, we focus on the exceptions by treating non-exceptions as censored random variables. In essence, this test provides a middle ground between the full distribution approach of the $L R_{\text {dist }}$ test and the frequency approach of the $L R_{u c}$ and $L R_{c c}$ tests.

As with the $L R_{d i s t}$ test, the empirical quantile series is transformed into standard normal $z_{m t+1}$ series. However, the $z_{m t+1}$ values are treated as censored normal random variables, where the censoring is tied to the desired coverage level of the VaR estimates. Thus, $z_{m t+1}$ is transformed into $\gamma_{m t+1}$ as follows:

$$
\gamma_{m t+1}=\left\{\begin{array}{l}
z_{m t+1} \quad \text { if } z_{m t+1}<\Phi^{-1}(\alpha) \\
0 \quad \text { if } z_{m t+1} \geq \Phi^{-1}(\alpha)
\end{array}\right.
$$

where $\Phi$ is the standard normal cumulative distribution function. The conditional likelihood function for the right-censored observations of $\gamma_{m t+1}=0$ (i.e., for non-exceptions) is

$$
f\left(\gamma_{m t+1} \mid z_{m t+1} \geq \Phi^{-1}(\alpha)\right)=1-\Phi\left(\frac{\Phi^{-1}(\alpha)-\mu_{m}}{\sigma_{m}}\right)
$$

where $\mu_{m}$ and $\sigma_{m}$ are respectively the unconditional mean and standard deviation of the $z_{m t+1}$ 
series. $^{21}$ The conditional likelihood function for $\gamma_{m t+1}=z_{m t+1}$ is that of a truncated normal distribution,

$$
f\left(\gamma_{m t+1} \mid z_{m t+1}<\Phi^{-1}(\alpha)\right)=\frac{\phi\left(\gamma_{m t+1}\right)}{\Phi\left(\frac{\Phi^{-1}(\alpha)-\mu_{m}}{\sigma_{m}}\right)} .
$$

where $\phi$ is the standard normal density function. Thus, the unconstrained conditional log-likelihood is

$$
\begin{aligned}
L_{m a g}\left(\mu_{m}, \sigma_{m}\right)= & \sum_{\gamma_{m t+1}=0} \log \left[1-\Phi\left(\frac{\Phi^{-1}(\alpha)-\mu_{m}}{\sigma_{m}}\right)\right] \\
& +\sum_{\gamma_{m t+1} \neq 0}-\frac{1}{2} \log \left(2 \pi \sigma_{m}^{2}\right)-\frac{\left(\gamma_{m t+1}-\mu_{m}\right)^{2}}{2 \sigma_{m}^{2}}-\log \left[\Phi\left(\frac{\Phi^{-1}(\alpha)-\mu_{m}}{\sigma_{m}}\right)\right] .
\end{aligned}
$$

If the VaR model generating the empirical quantiles is correct, the $\gamma_{m t+1}$ series should be identically distributed, and $\left(\mu_{m}, \sigma_{m}\right)$ should equal $(0,1)$. Thus, the relevant test statistic is

$$
L R_{m a g}=2\left[L_{m a g}\left(\mu_{m}, \sigma_{m}\right)-L_{m a g}(0,1)\right]
$$

which is asymptotically distributed $\chi^{2}(2)$.

\subsubsection{Regulatory Loss Function}

Under the 1996 Market Risk Amendment (MRA) to the Basel Capital Accord, regulatory capital for the trading positions (e.g. interest rate and foreign exchange positions) of commercial banks is set according to the banks' own internal VaR estimates. Given its actual use by market participants, the regulatory loss function implied in the MRA is a natural way to evaluate the relative performance of VaR estimates within an economic framework; see Lopez (1999b) for further discussion.

\footnotetext{
${ }^{21}$ Note that this test does not examine the autocorrelation coefficient $\rho_{m}$ discussed before, since the transformation $z_{m t+1}$ into the censored random variable $\gamma_{m t+1}$ disrupts the time sequence of the series. Thus, we only examine the two unconditional moments of the $z_{m t+1}$ series.
} 
The portfolio return in US dollars can expressed as

$$
\begin{aligned}
R_{p t+1} & =\ln \left(Y_{\mathrm{US} \$ t+1}\right)-\ln \left(Y_{\mathrm{US} \$ t}\right)+\ln \left(Y_{\mathrm{DM} t+1}\right)-\ln \left(Y_{\mathrm{DM} t}\right)+\ln \left(Y_{\mathrm{JY} t+1}\right)-\ln \left(Y_{\mathrm{JY} t}\right) \\
& =\ln \left(Y_{\mathrm{US} \$ t+1} Y_{\mathrm{DM} t+1} Y_{\mathrm{JY} t+1}\right)-\ln \left(Y_{\mathrm{US} \$ t} Y_{\mathrm{DM} t} Y_{\mathrm{JY} t}\right),
\end{aligned}
$$

and the portfolio value in US dollar terms is,

$$
\begin{aligned}
Y_{p t+1} & =Y_{p t} e^{R_{p t+1}} \\
& =Y_{\mathrm{US} \$ t+1} Y_{\mathrm{DM} t+1} Y_{\mathrm{JY} t+1}
\end{aligned}
$$

Replacing $R_{p t+1}$ with the desired $\mathrm{VaR}$ estimate, $\operatorname{VaR}_{m t}(\alpha)$, we generate what the portfolio value is expected to be at the lower $\alpha$ tail of the portfolio return distribution. Hence, to express the VaR estimate in dollar terms, we have

$$
\operatorname{VaR} \$_{m t}(\alpha)=Y_{p t}\left(1-e^{V a R_{m t}(\alpha)}\right)
$$

In basic operational terms, we need to choose a starting portfolio value and track it over the sample period in order to carry out this calculation. Note that we cannot standardize the portfolio values over time by simply dividing by the initial value. The path dependence of the portfolio value cannot be removed and must be accounted for in the calculations. The market-risk capital loss function is specified as

$$
\mathrm{MRC}_{m t}=\sqrt{10} \max \left[\operatorname{VaR} \$_{m t}(1 \%), \frac{S_{m t}}{60} \sum_{k=0}^{59} \operatorname{VaR} \$_{m t-k}(1 \%)\right]+S R_{m t}
$$

where $S_{m t}$ is the MRA's multiplication factor (i.e., from 3 to 4 depending on the number of exceptions over the past 250 days) and $S R_{m t}$ is a specific risk charge that does not have to do with the VaR modeling.

Under the current framework, $S_{m t} \geq 3$, and it is a step function that depends on the number of exceptions observed over the previous 250 trading days. ${ }^{22}$ The possible number of exceptions

\footnotetext{
${ }^{22}$ Note that the portfolio returns reported to the regulators, commonly referred to as the profit \& loss numbers, will usually not directly correspond to $R_{p t+1}$. The profit \& loss numbers are usually polluted by the presence of
} 
is divided into three zones. Within the green zone of four or fewer exceptions, a VaR model is deemed "acceptably accurate" to the regulators, and $S_{m t}$ remains at its minimum value of three. Within the yellow zone of five to nine exceptions, $S_{m t}$ increases incrementally with the number of exceptions ${ }^{23}$. Within the red zone of ten or more exceptions, the VaR model is deemed to be "inaccurate" for regulatory purposes, and $S_{m t}$ increases to its maximum value of four. The institution must also explicitly take steps to improve its risk management system. Thus, banks look to minimize exceptions (in order to minimize the multiplier) without reporting VaR estimates that are too large and raise the average term in the loss function.

\section{VaR Evaluation Results}

This section presents the forecast evaluation results of our VaR models. Tables 4 and 5 report, respectively, mean VaR estimates and the percentage of exceptions observed for each of the $60 \mathrm{VaR}$ models for the $1 \%, 5 \%, 10 \%$ and $25 \%$ quantiles over the entire out-of-sample period. These summary statistics are key components of the VaR evaluation results reported in Tables 6-13. Both the tables and the discussion below are framed with respect to the distributional assumption first and then with respect to the relative performance of the 15 models' second-moment specifications. Note that eleven of the models specify the multivariate dynamics of the conditional variance-covariance matrix and that four models only specify the dynamics of the portfolio's conditional variance.

Table 4 show the mean VaR estimates for our alternative models. The most striking feature is that the mean VaR estimates depends mainly on the VaR distributional assumption, rather than on the covariance matrix forecast. The average 1\% VaR estimates for the $\mathrm{t}$ - and generalized t-distributions lies outside the first percentile of the portfolio return distribution; see Table 1 . In contrast, the average 1\% VaR estimates under the normal distribution is greater than the first percentile of the portfolio return. The nonparametric mean VaR estimate at the $1 \%$ quantile is quite close the first percentile of the portfolio return for most covariance forecasts.

The observed frequency of exceptions reported in Table 5 confirms that the VaR normal estimates at the $1 \%$ quantile are too low, with the observed frequency of exceptions above $1 \%$ for all covariance matrix forecasts. However, at higher quantiles, the normal distribution tend to over-

customer fees and intraday trade results, which are not captured in standard VaR models.

${ }^{23}$ In the yellow zone, the multiplier values for five through nine exceptions are $3.4,3.5,3.75$ and 3.85 , respectively. 
estimate the VaR estimates. While the t-distribution VaR estimates are too conservative at all quantiles, the generalized t-distribution VaR estimates do not provide a clear pattern. The nonparametric distribution assumption VaR estimates perform reasonable well at all quantiles, but they show a tendency to generate VaR estimates that are too conservative.

\subsection{Unconditional and Conditional Coverage Tests}

Tables 6 and 7 report results for the unconditional $\left(L R_{u c}\right)$ and conditional coverage $\left(L R_{c c}\right)$ tests, respectively. Note that the $L R_{u c}$ and $L R_{c c}$ test results are qualitatively similar. VaR models based on the standard normal distributional assumption perform relatively well at the $1 \%$ quantile, in that only a few sets of VaR estimates fail the $L R_{u c}$ and $L R_{c c}$ tests at the $5 \%$ significance level. However, almost all fail at the higher quantiles $(5 \%, 10 \%$ and $25 \%)$. It is interesting to find that the standard normal distribution generates VaR estimates that perform well at such a low quantile, especially since portfolio returns are commonly found to be distributed non-normally, in particular with fatter tails. This result appears to be in line with those of Lucas (2000), who found that the upward bias in the estimated dispersion measure under such models appears to partially offset the neglected leptokurtosis.

VaR models based on the t-distribution assumption perform only modestly well for the $1 \%$ quantile, i.e. only about a third of the covariance matrix specifications do not reject the null hypotheses of the $L R_{u c}$ and $L R_{c c}$ tests. All specifications reject the null hypotheses at the higher quantiles. Similarly, the results for the VaR models based on the generalized t-distribution, indicate that most of the covariance matrix specifications fail at the lowest quantile, although they have some very limited success at the higher quantiles. This result suggests that the tail thickness of these distributional assumptions probably limits their use to just the lowest VaR coverage levels.

The nonparametric distributional results coverage test results provide evidence that these VaR estimates do relatively well across all four quantiles. For the $1 \%$ quantile, all the specifications do not reject the null hypotheses of correct unconditional and conditional coverage. While the performance for the $5 \%$ quantiles is relatively poor and similar to alternative distributional assumptions, the performance for the $10 \%$ and $25 \%$ quantiles is quite reasonable with non-rejections of the null hypotheses in about $50 \%$ and $60 \%$ of the cases, respectively.

Although we can conclude that the standard normal and the nonparametric distributional 
assumptions produce the best VaR estimates, inference regarding the relative forecast accuracy of the 11 covariance matrix specifications and 4 portfolio variance specifications is limited. As shown in Tables 6 and 7, no strong conclusions can be drawn across the forecasts using the normal or nonparametric distributional assumptions, specially at the $1 \%$ quantile. In addition, under the tdistribution and generalized t-distribution little inference is possible, though a little bit more than under the two alternative distributional assumptions.

The clearest result is that the best specification among the multivariate models seems to be the BEKK model, while the worst performers are the CCORR model and the GDC model, which is the most general one. There is no clear evidence that the level effect is important for VaR model performance. The simple covariance forecasts seem to present similar performance to the best multivariate models, in particular the exponentially- and equally-weighted using past 250 observations. Furthermore, univariate portfolio variance specifications that abstract from covariance matrix forecasts indicate that one can reasonably simplify the generation of VaR estimates in this way without sacrificing the accuracy of unconditional and conditional coverage.

In summary, these results indicate that the dominant factor in determining the relative accuracy of VaR estimates with respect to coverage is the distributional assumption, which it is consistent with the Lopez and Walter (2001) findings. The specifications of the portfolio variance, whether based on covariance matrix forecasts or univariate forecasts, appear to be of second-order importance. These results provide support for the common industry practice of using the simple portfolio variance specifications in generating VaR estimates. However, it does not completely explain why practitioners have generally settled on the standard normal distributional assumption. Although

it did perform well for the lower coverage levels that are usually of interest, the nonparametric distribution did better across the four quantiles examined.

\subsection{Dynamic Quantile Test}

Table 8 presents the results of the VaR estimates evaluation using the Dynamic Quantile test proposed by Engle and Manganelli (1999). The results are mainly consistent with the ones using the unconditional and conditional coverage tests. In fact, VaR models based on the standard normal and nonparametric distributional assumption perform relatively well at the $1 \%$ quantile. Under the non-parametric distributional assumption, all the specifications do not reject the null hypotheses 
of correct $1 \%$ VaR estimates, and the number of rejections under the standard normal is just two. Furthermore, the nonparametric distribution presents the best performance at higher quantiles. The VaR models based on the t-distribution and generalized t-distribution perform slightly better for the $1 \%$ VaR estimates than in the unconditional and conditional coverage tests, even though the performance is still clearly worse than for the alternative distributional assumptions.

\subsection{Distribution Forecast Test}

Table 9 contains the $L R_{\text {dist }}$ test results for the $60 \mathrm{VaR}$ models. These results again show that the standard normal and the nonparametric distributional assumptions generate VaR estimates that perform relatively well. The null hypothesis of correct conditional distributional form is not rejected in about half of the cases. For the entire distribution, the generalized-t distributional assumption performs equally well, suggesting that these models' quantiles closer to the median perform better than their tail quantiles. The t-distributional assumption again performs poorly.

These results provide further evidence that the distributional assumption appears to drive the VaR forecast evaluation results and that the portfolio variance specification is of secondary importance. Once again, inference on the relative performance of these models is possible, but limited. The clearest result is that the CCORR and GDC specifications are found to reject the null hypothesis in all cases. The BEKK and VECH specifications present the best performance among the multivariate models, but the level effect can again be dropped without affecting model accuracy. Furthermore, the basically equivalent performance of the multivariate covariance matrix specifications and the simple univariate forecasts, regardless of considering the individual portfolio components, confirms that one may simplify the VaR calculations without being penalized in terms of performance.

\subsection{Exception Magnitudes}

The previous tests do not take into account the magnitude of the exceptions, but only their frequency. In this section, we analyze model performance by considering the size of the loss when an exception occurs. Table 10 reports the mean exception; i.e., the average loss in excess of the VaR estimate conditional on the portfolio return being less than the VaR estimate. The mean exception is approximately $50 \%$ of the portfolio return standard deviation beyond the VaR estimate. As 
expected, the models based on the t-student distribution generate the lowest mean exceptions, but only at the $1 \%$ quantile. The models based on the normal distribution seem to have the largest mean exceptions at the $1 \%$ quantile, but at the $5 \%$ and higher quantiles, they have similar mean exceptions. The distributional assumption does not seem to play such a dominant role in terms of exception magnitude, as it did in terms of exception frequency.

The results of the $L R_{\text {mag }}$ tests for the four sets of VaR estimates using alternative distributional assumptions are reported in Table 11. Since this is a joint null hypothesis regarding the frequency of exceptions and their magnitudes, we expect that it would be rejected for the cases in which the VaR estimates rejected the binomial null hypothesis alone. This result occurs in about $96 \%$ of the cases (153 out of 160), but the bulk of the unexpected non-rejections of the joint null hypothesis (4 out of 7) occur for the 1\% VaR estimates, where the power of the binomial test is at its lowest. Overall, the two sets of test results were in agreement in $74 \%$ of the cases (178 out of 240 ), and the joint null hypothesis was rejected after the binomial null hypothesis was not rejected in another $23 \%$ of cases (55 of 240 ). Thus, the $L R_{\text {mag }}$ results are consistent with the $L R_{u c}$ results in $97 \%$ of the cases, which is quite a reasonable rate of cross-test correspondence.

The vast majority of the VaR estimates reject the null hypothesis that the magnitude of their VaR exceptions are consistent with the model, suggesting that the models are misspecified. In $90 \%$ of the cases (217 out of 240 ), the null hypothesis is rejected. Of the 23 cases in which the null hypothesis is not rejected, about $60 \%$ of the cases (14 out of 23 ) are based on the $1 \%$ VaR estimates.

Focusing first on the distributional assumptions underlying the VaR models, as before, the tdistributional assumption performs poorly, and in all of its 60 cases, the null hypothesis is rejected. The VaR estimates based on the other distributional assumptions perform equally well, although all of the cases using the standard normal distribution that perform well are for the $1 \%$ quantile.

Focusing on the different specifications of the portfolio variance forecasts, more inference across specifications using the $L R_{\text {mag }}$ test is possible because relatively few cases do not reject the null hypothesis. For the standard normal distribution, inference on specifications is the most difficult since half of the cases are found to be adequately specified. However, under the other two distributions, more inference is possible.

Under the generalized t-distribution, the BEKK, VECH and VECH-L specifications perform 
well, although the VECH and VECH-L specification does so at only the $1 \%$ quantile. However, the simple multivariate EWMA specification performs equally well at the $1 \%$ quantile. None of the other univariate specifications pass the $L R_{\text {mag }}$ hypothesis test. If we combine the results of the $L R_{u c}$ and the $L R_{m a g}$ tests, only the BEKK, VECH and VECH-L specifications perform well.

However, the clearest inference on the portfolio variance specification is possible under the nonparametric distribution. Of the multivariate specifications, only the BEKK-L and the E250 specifications perform reasonably well. Of the univariate specifications, the only one to perform well is the EWMA Portfolio specification, which does not reject the null hypothesis of correct specification for all quantiles. These results are not affected by combining the results of the $L R_{u c}$ and the $L R_{m a g}$ tests since they pass both of them at the $5 \%$ significance level. Thus, under the $L R_{\text {mag }}$ test, the VaR estimates based on the least complex model specification perform best. That is, the VaR estimates based on the simplest portfolio variance specification, the EWMA Portfolio model, and the simplest distributional assumption, the nonparametric distribution, do not reject the null hypothesis of correct model specification for all four quantiles of interest.

In summary, distributional assumptions also play an important role in the results of the $L R_{\text {mag }}$ test, as in the other hypothesis tests. However, these results allow more inference across the portfolio variance specifications, and the results indicate that the simplest VaR estimates perform best. The VaR estimates based on the EWMA Portfolio specification, which ignores the portfolio's components, and the nonparametric distribution, which has no simple closed form expression, performs best across the four quantiles of interest. This outcome presents further support and validation for the current use of these simple VaR estimates by financial institutions, if only because of the lower cost of generating these simple VaR estimates.

\subsection{Regulatory Loss Function}

As currently specified, the regulatory loss function is based on ten-day VaR estimates. However, since we are examining one-step-ahead portfolio variance forecasts, we evaluate one-day VaR estimates using this loss function as it is common practice in backtesting procedures. As this loss function does not correspond to a well-defined statistical test, we need to select a benchmark model against which we compare the others. Given the common use of the EWMA-Normal model in practice, we choose its capital charges to be the benchmark against which the other models' capital 
charges are compared.

Table 12 presents the percentage of the 2870 out-of-sample trading days for which the $\mathrm{MRC}_{m t+1}$ capital charges for the EWMA-Normal model are less than those of the other 59 VaR models. This model clearly has lower capital requirements than all of the portfolio variance specifications under the t-distribution and generalized-t distribution with the EWMA-Normal performing better than these alternatives more than $75 \%$ of the time. The only VaR estimates that generates smaller capital charges than those of the EWMA-Normal model for more than $50 \%$ of the trading days are those of the EWMA Portfolio model under the normal distribution (EWMA Portfolio-Normal). Note that the EWMA Portfolio model completely ignores the covariance matrix dynamics of the portfolios' component securities.

To more carefully examine these regulatory loss function results, we examine the differences between the capital charges for the EWMA-Normal model and the other models using the DieboldMariano test statistic. The null hypothesis that we investigate is whether the mean difference between the two sets of capital charges is equal to zero. If we do not reject the null hypothesis, then the alternative model does not perform worse than the EWMA-Normal model. If we reject the null hypothesis and the mean difference is negative, then the EWMA-Normal model and its VaR estimates perform better because they generate lower capital charges on average. If we reject the null hypothesis and the mean difference is positive, then the alternative model and its VaR estimates perform better.

Table 12 also present the p-values for the Diebold-Mariano statistics. In about $80 \%$ of the cases (47 of 59 cases), we reject the null hypothesis in favor of the EWMA-Normal model. We reject all the alternative $\mathrm{VaR}$ models based on the t-distribution and generalized-t distributional assumption. For the nonparametric distribution, we do not reject the null hypothesis for only the BEKK-L specification. For the normal distribution, 11 portfolio variance specifications do not reject the null hypothesis, indicating that they perform as well as the EWMA-Normal model under this regulatory loss function.

The most noteworthy case is the EWMA Portfolio-Normal distribution with a p-value of almost $100 \%$, which implies that the capital charges from this model are, on average, significantly lower than those for the benchmark model. In fact, when the EWMA Portfolio-Normal model is treated as the benchmark as shown in Table 13, we reject the null hypothesis in all but four cases: BEKK-L, 
E250, Eall and E250 Portfolio, all cases under the normal distribution. This implies that these VaR models perform as well as the EWMA Portfolio-Normal model under the regulatory loss function, but they generate higher capital charges in more than $60 \%$ of the trading days. However, the EWMA Portfolio-Normal model is much easier to estimate than the other models. Hence, under this loss function, we can clearly state that this simple practitioner model that ignores the covariance matrix dynamics of the portfolio's component securities and the fat-tailed nature of the portfolio returns is the best performing model to use when the objective is to minimize the regulatory capital requirements.

\section{Conclusion}

We examine VaR estimates for an international interest rate portfolio using a variety of multivariate volatility models, ranging from naive averages to standard time-series models. The question of interest is whether the most complex covariance models provide improved out-of-sample forecasts for use in VaR analysis in the case of an international interest rate portfolio.

We find that covariance matrix forecasts generated from models that incorporate interest-rate level effect perform best under statistical loss functions, such as mean-squared error. Within a VaR framework, the relative performance of covariance matrix forecasts depends greatly on the VaR models' distributional assumptions. Of the forecasts examined, simple specifications, such

as exponentially-weighted moving averages of past observations, perform best with regard to the magnitude of VaR exceptions and regulatory capital requirements. Our results provide empirical support for the commonly-used VaR models based on simple covariance matrix forecasts and distributional assumptions, such as the standard normal. Moreover, portfolio variance forecasts directly based on the portfolio returns, i.e. that ignore the covariance matrix dynamics of the portfolio's component securities, the fat-tailed nature of the portfolio returns and the current portfolio composition, present the best performance when the objective is to minimize the regulatory capital requirements.

Overall, our results are consistent with those of Lopez and Walter (2001) and Berkowitz and O'Brien (2002) for foreign-exchange rate portfolios and US commercial bank trading portfolios, respectively. Also, note that this result closely parallels that of Beltratti and Morana (1999), who 
found that volatility forecasts from more complex models are fairly similar to those of simpler GARCH models. They conclude that there is validity in the use of computationally simple models for forecasting volatility. Similarly, Christoffersen and Jacobs (2002) found that in contrast to inference based on stock returns, an objective function based on pricing stock options favors a relatively parsimonious model. Hence, our results are in line with recent other findings that for economic purposes, as expressed via economic loss functions associated with option pricing and risk management, volatility forecasts from simpler models are found to outperform the forecasts from more complex models.

The reasons for these findings range from the issue of parameter uncertainty, as per Skintzi, Skiadopoulos, and Refenes (2003), to overfitting the data in-sample to the sensitivity (i.e., the first derivative) of relevant economic loss functions to the volatility forecast inputs. An interesting paper that addresses this question more directly is Lucas (2000), who finds that VaR models based on simple measures of portfolio variance and the normal distribution generate smaller discrepancies between actual and postulated VaR estimates than more sophisticated VaR models. He argues that this outcome is based on offsetting biases in the variance and VaR estimates of simple models that cannot be captured by more sophisticated models that attempt to capture the actual (but unknown) degree of leptokurtosis in the portfolio returns. The reasons for these parallel results are worth further study. 


\section{References}

Alexander, C. O., and C. T. Leigh, 1997, On the covariance matrices used in value-at-risk models, Journal of Derivatives 4, 50-62.

Amin, K., and V. Ng, 1997, Inferring future volatility from the information in implied volatility in eurodollar options: A new approach, Review of Financial Studies 10, 333-367.

Andersen, T., and T. Bollerslev, 1998, Answering the skeptics: Yes, standard volatility models do provide accurate forecasts, International Economic Review 39, 885-905.

Bali, T., 2003, Modeling the stochastic behavior of short-term interest rates: Pricing implications for discount bonds, Journal of Banking and Finance 27, 201-228.

Beltratti, A., and C. Morana, 1999, Computing value-at-risk with high frequency data, Journal of Empirical Finance 6, 431-455.

Berkowitz, J., 2001, Testing density forecasts with applications to risk management, Journal of Business and Economic Statistics 19, 465-474.

Berkowitz, J., and J. O’Brien, 2002, How accurate are value-at-risk models at commercial banks?, Journal of Finance 57, 1093-1111.

Bollerslev, T., 1990, Modelling the coherence in short-run nominal exchange rates: A multivariate generalized ARCH model, Review of Economics and Statistics 72, 498-505.

Bollerslev, T., R. Engle, and J. Wooldridge, 1988, A capital asset pricing model with time-varying covariances, Journal of Political Economy 96, 116-131.

Bollerslev, T., and J. M. Wooldridge, 1992, Quasi-maximum likelihood estimation and inference in dynamic models in time-vaying covariances, Econometric Reviews 11, 143-172.

Brenner, R. J., R. Harjes, and K. Kroner, 1996, Another look at models of the short-term interest rate, Journal of Financial and Quantitative Analysis 31, 85-107. 
Campa, J., and K. Chang, 1998, The forecasting ability of correlations implied in foreign exhange options, Journal of International Money and Finance 17, 855-880.

Cecchetti, S., R. Cumby, and S. Figlewski, 1988, Estimation of the optimal futures hedge, Review of Economics and Statistics 623-630.

Chan, K., A. Karolyi, F. Longstaff, and A. Sanders, 1992, An empirical comparison of alternative models of short term interest rates, Journal of Finance 47, 1209-1227.

Chapman, D., J. Long, and N. Pearson, 1999, Using proxies for the short rate: When are three months like an instant?, Review of Financial Studies 12, 763-806.

Christoffersen, P., 1998, Evaluating interval forecasts, International Economic Review 39, 841-862.

Christoffersen, P., and K. Jacobs, 2002, Which volatility model for option valuation?, working paper, McGill University.

Christoffersen, P., and D. Pelletier, 2003, Backtesting value at risk: A duration-based approach, working paper, McGill University.

Crnkovic, C., and J. Drachman, 1996, Quality control, Risk 9, 139-143.

Dave, R., and G. Stahl, 1996, On the accuracy of VaR estimates based on the variance-covariance approach, working paper, German Federal Banking Supervisory Office.

Day, T., and C. Lewis, 1992, Stock market volatility and the information content of stock index options, Journal of Econometrics 52, 267-287.

Diebold, F., T. Gunther, and A. Tay, 1998, Evaluating density forecasts with applications to financial risk management, International Economic Review 39, 863-883.

Diebold, F., and R. Mariano, 1995, Comparing predictice accuracy, Journal of Business and Economic Statistics 13, 253-263.

Duffie, D., and K. Singleton, 1997, An econometric model of the term structure of interest-rate swap yields, Journal of Finance 52, 1287-1321.

Ederington, L., and W. Guan, 1999, Forecasting volatility, working paper, University of Oklahoma. 
Engle, R., 2002, Dynamic conditional correlation: A simple class of multivariate generalized autoregressive conditional heteroskedasticity models, Journal of Business and Economic Statistics 20, 339-350.

Engle, R., and G. Gonzalez-Rivera, 1991, Semiparametric ARCH models, Journal of Business and Economic Statistics 9, 345-359.

Engle, R., A. Kane, and J. Noh, 1997, Index-options pricing with stochastic volatility and the value of accurate variance forecasts, Review of Derivatives Research 1, 139-157.

Engle, R., and K. Kroner, 1995, Multivariate simultaneous generalized ARCH, Econometric Theory $11,122-150$.

Engle, R., and S. Manganelli, 1999, CAViaR: Conditional autoregressive value at risk by regression quantiles, working paper, University of California - San Diego.

Engle, R., and V. Ng, 1993, Measuring and testing the impact of news on volatility, Journal of Finance 48, 987-1007.

Ferreira, M. A., 2001, Forecasting the comovements of spot interest rates, Journal of International Money and Finance, forthcoming.

Figlewski, S., 1997, Forecasting volatility, Financial Markets, Institutions and Instruments 6.

Gibson, M., and B. Boyer, 1998, Evaluating forecasts of correlation using option pricing, Journal of Derivatives 18-38.

Glosten, L., R. Jagannathan, and D. Runkle, 1993, On the relation between the expected value and the volatility of the nominal excess return on stocks, Journal of Finance 48, 1779-1801.

Hendricks, D., 1996, Evaluation of value-at-risk models using historical data, Economic Policy Review 2, 39-69.

Jackson, P., D. Maude, and W. Perraudin, 1997, Bank capital and value at risk, Journal of Derivatives 4, 73-90.

Kroner, K., and V. Ng, 1998, Modeling asymmetric comovements of asset returns, Review of Financial Studies 11, 817-844. 
Kupiec, P., 1995, Techniques for verifying the accuracy of risk measurement models, Journal of Derivatives 3, 73-84.

Lopez, J., 1999a, Methods for evaluating value-at-risk estimates, Federal Reserve Bank of San Francisco Economic Review 2, 3-15.

Lopez, J., 1999b, Regulatory evaluation of value-at-risk models, Journal of Risk 1, 37-64.

Lopez, J., 2001, Evaluating the predictive accuracy of volatility models, Journal of Forecasting 20, $87-109$.

Lopez, J., and C. Walter, 2001, Evaluating covariance matrix forecasts in a value-at-risk framework, Journal of Risk 3, 69-98.

Lucas, A., 2000, A note on optimal estimation from a risk-management perspective under possibly misspecified tail behavior, Journal of Business and Economic Statistics 18, 31-39.

Pagan, A., and G. Schwert, 1990, Alternative models for conditional stock volatility, Journal of Econometrics 45, 267-290.

Poomimars, P., J. Cadle, and M. Theobald, 2001, Dynamic asset allocation with time-varying variance-covariance structures, working paper, University of Birmingham.

Skintzi, V.D., G. Skiadopoulos, and A. P. N. Refenes, 2003, The effect of mis-estimating correlation on value-at-risk, working paper, Financial Options Research Center, University of Warwick.

Vlaar, P., 2000, Value at risk models for dutch bond portfolios, Journal of Banking and Finance $24,1131-1154$.

Walter, C., and J. Lopez, 2000, Is implied correlation worth calculating? evidence from foreign exchange options, Journal of Derivatives 7, 65-82.

West, K., and D. Cho, 1995, The predictive ability of several models of exchange rate volatility, Journal of Econometrics 69, 367-391. 
Table 1: Summary Statistics of Interest and Exchange Rates

\begin{tabular}{|c|c|c|c|c|c|c|}
\hline & $\begin{array}{c}\text { US\$ } \\
\text { Interest Rate }\end{array}$ & $\begin{array}{c}\mathrm{DM} \\
\text { Interest Rate }\end{array}$ & $\begin{array}{c}\text { JY } \\
\text { Interest Rate }\end{array}$ & $\begin{array}{l}\text { DM/US\$ } \\
\text { FX Rate }\end{array}$ & $\begin{array}{l}\text { JY/US\$ } \\
\text { FX Rate }\end{array}$ & $\begin{array}{c}\text { Portfolio } \\
\text { Return }\end{array}$ \\
\hline \multicolumn{7}{|c|}{ Panel A: $\varepsilon_{t}$} \\
\hline Mean & -0.001 & 0.000 & 0.000 & 0.002 & -0.010 & 0.008 \\
\hline Standard Deviation & 0.173 & 0.085 & 0.114 & 0.670 & 0.695 & 1.213 \\
\hline Skewness & -0.207 & 0.320 & 0.352 & -0.261 & -0.664 & 0.418 \\
\hline Kurtosis & 28.85 & 18.53 & 48.91 & 2.770 & 5.055 & 3.109 \\
\hline Minimum & -2.375 & -1.000 & -1.813 & -5.785 & -5.835 & -6.046 \\
\hline 1-th Percentile & -0.031 & -0.016 & -0.004 & -0.349 & -0.334 & -0.661 \\
\hline Median & 0.000 & 0.000 & 0.000 & 0.000 & 0.000 & -0.004 \\
\hline 99-th Percentile & 0.034 & 0.031 & 0.004 & 0.364 & 0.360 & 0.639 \\
\hline Maximum & 2.500 & 1.125 & 1.625 & 3.037 & 3.385 & 10.725 \\
\hline \multirow[t]{2}{*}{$Q(10)$} & 489.6 & 31.73 & 65.16 & 13.17 & 18.63 & 13.48 \\
\hline & {$[0.000]$} & {$[0.000]$} & {$[0.000]$} & {$[0.214]$} & {$[0.045]$} & [0.198] \\
\hline \multirow[t]{2}{*}{$Q(30)$} & 1146.4 & 88.58 & 217.6 & 50.37 & 48.62 & 39.09 \\
\hline & {$[0.000]$} & {$[0.000]$} & {$[0.000]$} & {$[0.011]$} & {$[0.017]$} & {$[0.124]$} \\
\hline \multirow[t]{2}{*}{$Q(50)$} & 1789.1 & 125.1 & 332.0 & 74.81 & 63.97 & 56.91 \\
\hline & {$[0.000]$} & {$[0.000]$} & {$[0.000]$} & {$[0.013]$} & {$[0.089]$} & [0.233] \\
\hline \multicolumn{7}{|c|}{ Panel B: $\varepsilon_{t}^{2}$} \\
\hline Mean & 0.030 & 0.007 & 0.013 & 0.449 & 0.483 & 1.472 \\
\hline Standard Deviation & 0.166 & 0.032 & 0.093 & 0.980 & 1.285 & 3.330 \\
\hline Skewness & 19.79 & 20.24 & 19.23 & 9.893 & 10.79 & 10.70 \\
\hline Kurtosis & 605.0 & 619.6 & 494.4 & 241.6 & 195.3 & 262.1 \\
\hline Minimum & 0.000 & 0.000 & 0.000 & 0.000 & 0.000 & 0.000 \\
\hline 1-th Percentile & 0.000 & 0.000 & 0.000 & 0.024 & 0.020 & 0.078 \\
\hline Median & 0.001 & 0.001 & 0.000 & 0.128 & 0.122 & 0.426 \\
\hline 99-th Percentile & 0.004 & 0.004 & 0.004 & 0.469 & 0.449 & 1.506 \\
\hline Maximum & 6.250 & 1.266 & 3.285 & 33.47 & 34.05 & 14.16 \\
\hline \multirow[t]{2}{*}{$Q(10)$} & 1851.2 & 2417.5 & 2773.2 & 266.33 & 415.89 & 153.1 \\
\hline & {$[0.000]$} & {$[0.000]$} & {$[0.000]$} & {$[0.000]$} & {$[0.000]$} & {$[0.000]$} \\
\hline \multirow[t]{2}{*}{$Q(30)$} & 4700.5 & 2889.9 & 4958.3 & 519.5 & 663.0 & 283.4 \\
\hline & {$[0.000]$} & {$[0.000]$} & [0.000] & {$[0.000]$} & {$[0.000]$} & {$[0.000]$} \\
\hline \multirow[t]{2}{*}{$Q(50)$} & 6458.3 & 3221.2 & 6684.1 & 590.8 & 715.8 & 313.9 \\
\hline & {$[0.000]$} & {$[0.000]$} & [0.000] & {$[0.000]$} & {$[0.000]$} & {$[0.000]$} \\
\hline
\end{tabular}

This table reports summary statistics on daily interest rates changes and foreign exchage rates geometric returns. $Q(k)$ is the Ljung-Box test statistics of order $k$ with p-values in brackets. 
Table 2: Out-of-Sample RMSPE for Variances Forecasts

\begin{tabular}{lllllll}
\hline \hline & \multicolumn{3}{c}{ Interest Rate Variance } & \multicolumn{2}{c}{ FX Rate Variance } & \multicolumn{1}{c}{ Portfolio } \\
\cline { 2 - 6 } & \multicolumn{1}{c}{ US $\$$} & DM & \multicolumn{1}{c}{ JY } & DM/US\$ & JY/US $\$$ & Variance $\left(\times 10^{2}\right)$ \\
\hline BEKK & $0.01467^{* *}$ & 0.01392 & $0.01009^{*}$ & $\mathbf{0 . 8 5 3 9 7}$ & 1.34429 & 0.02876 \\
BEKK-L & 0.01462 & $0.01534^{* * *}$ & 0.00991 & $\underline{\mathbf{0 . 8 5 3 7 0}}$ & 1.34335 & 0.02873 \\
CCORR & $0.01477^{* * *}$ & 0.01385 & $0.01019^{*}$ & 0.85635 & $\mathbf{1 . 3 3 9 4 1}$ & 0.02883 \\
CCORR-L & $\underline{\mathbf{0 . 0 1 4 5 3}}$ & 0.01369 & 0.01005 & 0.85636 & $\mathbf{1 . 3 3 9 4 2}$ & 0.02883 \\
GDC & $0.01480^{* * *}$ & $\underline{\mathbf{0 . 0 1 3 6 8}}$ & $0.01049^{* *}$ & $0.85714^{* *}$ & 1.34031 & 0.02874 \\
GDC-L & $0.01467^{* * *}$ & 0.01379 & $0.01037^{* *}$ & $0.85626^{* *}$ & 1.34042 & 0.02875 \\
VECH & $0.01475^{* * *}$ & 0.01381 & $0.01017^{*}$ & 0.85568 & 1.34138 & $\mathbf{0 . 0 2 8 6 8}$ \\
VECH-L & $\mathbf{0 . 0 1 4 5 5 * * *}$ & $\underline{\mathbf{0 . 0 1 3 6 8}}$ & 0.01002 & 0.85572 & 1.34139 & $\mathbf{0 . 0 2 8 6 8}$ \\
E250 & 0.01476 & 0.01423 & $\underline{\mathbf{0 . 0 0 9 8 0}}$ & $0.86828^{* * *}$ & $1.37766^{* *}$ & $0.02930^{* * *}$ \\
Eall & $0.04049^{* * *}$ & $0.01519^{* * *}$ & $0.01893^{* * *}$ & $0.87222^{* * *}$ & $1.38265^{*}$ & $0.02918^{* * *}$ \\
EWMA & $0.01461^{* * *}$ & 0.01395 & $\mathbf{0 . 0 0 9 8 7}$ & $0.85694^{*}$ & 1.34830 & 0.02880 \\
E250 Port & - & - & - & - & - & $0.02930^{* * *}$ \\
Eall Port & - & - & - & - & - & $0.02921^{* * *}$ \\
EWMA Port & - & - & - & - & - & 0.02869 \\
GARCH Port & - & - & - & - & - & 0.02881 \\
\hline \hline
\end{tabular}

This table presents RMSPE for the daily interest rate changes variance and foreign exchange rate geometric returns variance using alternative model specifications. The minimum value in each column is in bold and underlined, and the second smallest value is just in bold. ${ }^{* * *}$ denotes significance at the $1 \%$ level, ${ }^{* *}$ at the $5 \%$ level and * at the $10 \%$ level of the Diebold-Mariano test statistic, which test the null hypothesis of equal loss functions values between the forecast and the minimizing forescat. 
Table 3: Out-of-Sample RMSPE for Covariances Forecasts

\begin{tabular}{|c|c|c|c|c|c|c|c|c|c|c|}
\hline & \multicolumn{3}{|c|}{ Interest Rates Covariance } & \multicolumn{6}{|c|}{ Covariances between Interest Rates and FX Rates } & \multirow{3}{*}{$\begin{array}{c}\text { FX Rates } \\
\text { Covariance } \\
\text { DM/US\$ } \\
\text { JY/US\$ }\end{array}$} \\
\hline & US\$ & $\mathrm{US \$}$ & DM & US\$ & US\$ & $\mathrm{DM}$ & $\mathrm{DM}$ & JY & JY & \\
\hline & $\mathrm{DM}$ & JY & JY & $\mathrm{DM} / \mathrm{US} \$$ & JY/US\$ & $\mathrm{DM} / \mathrm{US} \$$ & JY/US\$ & $\mathrm{DM} / \mathrm{US} \$$ & JY/US\$ & \\
\hline BEKK & $0.00762^{*}$ & $0.00356^{* * *}$ & $0.00391^{* * *}$ & $0.05401^{* *}$ & $0.04159^{* * *}$ & $0.05799^{*}$ & $0.04487^{* *}$ & $0.03500^{* * *}$ & $0.03802^{* *}$ & $\underline{0.64996}$ \\
\hline BEKK-L & $\underline{0.00750}$ & $0.00345^{* * *}$ & $0.00395^{* * *}$ & 0.05332 & $\underline{0.04078}$ & 0.05645 & $0.04469^{*}$ & $0.03486^{* * *}$ & $0.03796^{* * *}$ & $\overline{0.65004}$ \\
\hline CCORR & 0.00754 & $0.00343^{* *}$ & $0.00379^{* *}$ & 0.05344 & 0.04095 & $0.05756^{*}$ & 0.04473 & $0.03424^{* *}$ & $0.03750^{* * *}$ & $0.66641^{* * *}$ \\
\hline CCORR-L & 0.00751 & $0.00342^{*}$ & $0.00379^{* *}$ & 0.05340 & 0.04087 & $0.05764^{*}$ & 0.04472 & $\underline{0.03422}$ & $\underline{0.03745}$ & $0.66641^{* * *}$ \\
\hline GDC & $0.00759^{*}$ & $0.00346^{* * *}$ & $0.00381^{* * *}$ & 0.05324 & $0.04130^{* *}$ & $0.05771^{*}$ & 0.04476 & $\overline{0.03453^{* * *}}$ & $\overline{0.03759^{* * *} *}$ & $0.65609^{* * *}$ \\
\hline GDC-L & $0.00759^{*}$ & $0.00345^{* * *}$ & $0.00382^{* * *}$ & 0.05334 & $0.04117^{* *}$ & $0.05787^{*}$ & $0.04487^{*}$ & $0.03455^{* * *}$ & $0.03759^{* * *}$ & $0.65486^{* * *}$ \\
\hline VECH & 0.00753 & $0.00343^{* * *}$ & $0.00378^{* *}$ & $\underline{0.05323}$ & 0.04084 & 0.05593 & $0.04461^{* *}$ & 0.03425 & 0.03752 & $0.65365^{*}$ \\
\hline VECH-L & 0.00751 & 0.00340 & $0.00378^{* * *}$ & 0.05324 & 0.04082 & 0.05598 & $0.04460^{* *}$ & 0.03426 & 0.03752 & $0.65377^{*}$ \\
\hline E250 & $\underline{0.00750}$ & $\underline{0.00339}$ & $\underline{0.00376}$ & 0.05356 & 0.04080 & 0.05579 & $\underline{0.04434}$ & 0.03428 & 0.03746 & $0.65753^{* * *}$ \\
\hline Eall & $0.00846^{* * *}$ & $0.00388^{* * *}$ & $0.00402^{* * *}$ & $0.06050^{* * *}$ & $0.04476^{* * *}$ & 0.05666 & $0.04488^{* * *}$ & $0.03439^{* * *}$ & $0.03802^{* * *}$ & $0.66157^{* * *}$ \\
\hline EWMA & 0.00754 & $0.00343^{* *}$ & $0.00382^{* * *}$ & 0.05355 & $0.04111^{* *}$ & $\underline{0.05507}$ & $0.04500^{* *}$ & $0.03474^{* * *}$ & $0.03788^{*}$ & 0.65214 \\
\hline
\end{tabular}

This table presents RMSPE for the daily interest rate changes and foreign exchange rate geometric returns covariances using alternative model specifications. The minimum value in each column is in bold and underlined, and the second smallest value is just in bold. ${ }^{* * *}$ denotes significance at the $1 \%$ level, ${ }^{* *}$ at the $5 \%$ level and ${ }^{*}$ at the $10 \%$ level of the Diebold-Mariano test statistic, which test the null hypothesis of equal loss functions values between the forecast and the minimizing forescat. 
Table 4: Mean Value-at-Risk

\begin{tabular}{|c|c|c|c|c|c|c|c|c|c|c|c|c|c|c|c|c|}
\hline & \multicolumn{4}{|c|}{ Normal distribution } & \multicolumn{4}{|c|}{ t-distribution } & \multicolumn{4}{|c|}{ Generalized t-distribution } & \multicolumn{4}{|c|}{ Nonparametric distribution } \\
\hline & $1 \%$ & $5 \%$ & $10 \%$ & $25 \%$ & $1 \%$ & $5 \%$ & $10 \%$ & $25 \%$ & $1 \%$ & $5 \%$ & $10 \%$ & $25 \%$ & $1 \%$ & $5 \%$ & $10 \%$ & $25 \%$ \\
\hline BEKK & -2.704 & -1.915 & -1.494 & -0.790 & -3.158 & -2.090 & -1.589 & -0.817 & -3.257 & -1.568 & -1.090 & -0.527 & -2.952 & -1.888 & -1.390 & -0.681 \\
\hline BEKK-L & -2.721 & -1.928 & -1.505 & -0.799 & -3.177 & -2.104 & -1.601 & -0.826 & -3.015 & -1.461 & -1.025 & -0.509 & -2.802 & -1.882 & -1.393 & -0.688 \\
\hline CCORR & -2.879 & -2.040 & -1.592 & -0.844 & -3.419 & -2.246 & -1.703 & -0.875 & -4.047 & -1.444 & -1.395 & -0.683 & -3.244 & -2.092 & -1.530 & -0.759 \\
\hline CCORR-L & -2.880 & -2.040 & -1.592 & -0.843 & -3.419 & -2.246 & -1.703 & -0.874 & -4.398 & -2.216 & -1.539 & -0.738 & -3.227 & -2.091 & -1.525 & -0.758 \\
\hline GDC & -2.827 & -2.000 & -1.559 & -0.822 & -3.358 & -2.203 & -1.669 & -0.853 & -3.406 & -1.637 & -1.135 & -0.546 & -3.164 & -2.022 & -1.484 & -0.734 \\
\hline GDC-L & -2.806 & -1.988 & -1.552 & -0.823 & -3.332 & -2.190 & -1.661 & -0.854 & -3.379 & -1.629 & -1.133 & -0.550 & -3.139 & -2.034 & -1.498 & -0.738 \\
\hline VECH & -2.761 & -1.955 & -1.526 & -0.808 & -3.224 & -2.134 & -1.623 & -0.836 & -3.059 & -1.480 & -1.038 & -0.514 & -3.040 & -1.956 & -1.406 & -0.697 \\
\hline VECH-L & -2.768 & -1.961 & -1.530 & -0.811 & -3.232 & -2.140 & -1.627 & -0.838 & -3.067 & -1.484 & -1.041 & -0.516 & -3.048 & -1.961 & -1.412 & -0.699 \\
\hline E250 & -2.718 & -1.922 & -1.497 & -0.788 & -3.296 & -2.142 & -1.616 & -0.821 & -4.157 & -2.089 & -1.447 & -0.689 & -2.906 & -1.967 & -1.430 & -0.683 \\
\hline Eall & -2.793 & -1.975 & -1.538 & -0.810 & -3.477 & -2.232 & -1.677 & -0.848 & -4.663 & -2.431 & -1.681 & -0.795 & -3.141 & -2.040 & -1.488 & -0.681 \\
\hline EWMA & -2.618 & -1.851 & -1.442 & -0.759 & -3.259 & -2.092 & -1.572 & -0.795 & -4.371 & -2.279 & -1.576 & -0.746 & -3.155 & -1.950 & -1.400 & -0.626 \\
\hline E250 Port & -2.724 & -1.926 & -1.500 & -0.790 & -3.182 & -2.103 & -1.596 & -0.817 & -3.019 & -1.455 & -1.017 & -0.498 & -2.868 & -1.911 & -1.378 & -0.653 \\
\hline Eall Port & -2.869 & -2.028 & -1.580 & -0.832 & -3.408 & -2.235 & -1.692 & -0.863 & -4.387 & -2.205 & -1.527 & -0.727 & -3.130 & -2.027 & -1.493 & -0.719 \\
\hline EWMA Port & -2.622 & -1.854 & -1.444 & -0.760 & -3.180 & -2.066 & -1.559 & -0.792 & -4.009 & -2.015 & -1.396 & -0.665 & -3.030 & -1.900 & -1.388 & -0.665 \\
\hline GARCH Port & -2.800 & -1.979 & -1.542 & -0.812 & -3.226 & -2.145 & -1.632 & -0.837 & -3.104 & -1.496 & -1.045 & -0.512 & -3.002 & -1.986 & -1.440 & -0.708 \\
\hline
\end{tabular}

This table shows mean VaR estimates in percentage for the alternative VaR models under the 1\%, $5 \%$, $10 \%$ and $25 \%$ significance levels. 
Table 5: Observed Frequency of Exceptions

\begin{tabular}{|c|c|c|c|c|c|c|c|c|c|c|c|c|c|c|c|c|}
\hline & \multicolumn{4}{|c|}{ Normal distribution } & \multicolumn{4}{|c|}{ t-distribution } & \multicolumn{4}{|c|}{ Generalized t-distribution } & \multicolumn{4}{|c|}{ Nonparametric distrib. } \\
\hline & $1 \%$ & $5 \%$ & $10 \%$ & $25 \%$ & $1 \%$ & $5 \%$ & $10 \%$ & $25 \%$ & $1 \%$ & $5 \%$ & $10 \%$ & $25 \%$ & $1 \%$ & $5 \%$ & $10 \%$ & $25 \%$ \\
\hline BEKK & 1.29 & 4.25 & 7.94 & 21.56 & 0.70 & 3.10 & 6.58 & 20.77 & 0.59 & 6.93 & 14.04 & 29.69 & 0.87 & 4.39 & 9.02 & 24.39 \\
\hline BEKK-L & 1.18 & 4.18 & 7.87 & 21.25 & 0.66 & 3.07 & 6.59 & 20.59 & 0.77 & 8.22 & 15.44 & 30.28 & 0.98 & 4.49 & 8.95 & 24.29 \\
\hline CCORR & 1.21 & 3.45 & 6.76 & 20.21 & 0.49 & 2.47 & 5.75 & 19.37 & 0.17 & 8.57 & 9.30 & 24.91 & 0.66 & 3.17 & 7.70 & 22.26 \\
\hline CCORR-L & 1.22 & 3.45 & 6.76 & 20.21 & 0.49 & 2.47 & 5.75 & 19.37 & 0.10 & 2.65 & 7.53 & 22.96 & 0.70 & 3.17 & 7.74 & 22.37 \\
\hline GDC & 1.08 & 3.66 & 7.28 & 20.77 & 0.42 & 2.72 & 5.92 & 20.07 & 0.38 & 6.20 & 13.45 & 29.09 & 0.66 & 3.55 & 8.12 & 23.17 \\
\hline GDC-L & 1.08 & 3.73 & 7.28 & 20.73 & 0.49 & 2.79 & 6.03 & 20.03 & 0.42 & 6.24 & 13.48 & 28.71 & 0.70 & 3.41 & 8.01 & 22.96 \\
\hline VECH & 1.15 & 4.08 & 7.63 & 21.01 & 0.59 & 3.10 & 6.59 & 20.45 & 0.77 & 8.15 & 15.33 & 30.45 & 0.80 & 4.08 & 9.02 & 24.36 \\
\hline VECH-L & 1.15 & 4.01 & 7.60 & 20.94 & 0.59 & 3.07 & 6.59 & 20.34 & 0.77 & 8.12 & 15.26 & 30.21 & 0.77 & 4.01 & 8.89 & 24.29 \\
\hline E250 & 1.57 & 4.36 & 8.36 & 21.01 & 0.66 & 3.27 & 7.18 & 20.24 & 0.14 & 3.48 & 9.02 & 24.29 & 1.32 & 4.22 & 9.16 & 24.56 \\
\hline Eall & 1.36 & 4.04 & 7.70 & 20.59 & 0.52 & 2.72 & 5.99 & 19.79 & 0.07 & 2.09 & 5.99 & 20.98 & 0.73 & 3.55 & 8.22 & 23.83 \\
\hline EWMA & 1.71 & 5.02 & 8.85 & 22.86 & 0.63 & 3.52 & 7.49 & 21.32 & 0.10 & 2.75 & 7.49 & 23.55 & 0.73 & 4.32 & 9.34 & 26.69 \\
\hline E250 Port & 1.53 & 4.36 & 8.26 & 20.98 & 0.84 & 3.38 & 7.39 & 20.35 & 1.15 & 8.89 & 16.03 & 30.38 & 1.36 & 4.34 & 9.79 & 25.54 \\
\hline Eall Port & 1.25 & 3.69 & 7.04 & 20.03 & 0.56 & 2.72 & 5.75 & 19.48 & 0.10 & 2.96 & 7.77 & 22.40 & 0.77 & 3.69 & 8.08 & 22.61 \\
\hline EWMA Port & 1.57 & 5.09 & 8.95 & 22.89 & 0.77 & 3.69 & 7.80 & 21.50 & 0.21 & 3.97 & 9.51 & 25.68 & 0.84 & 4.60 & 9.51 & 25.64 \\
\hline GARCH Port & 1.11 & 3.83 & 7.35 & 20.87 & 0.59 & 2.86 & 6.27 & 20.14 & 0.70 & 7.94 & 14.98 & 30.00 & 0.87 & 3.83 & 8.50 & 23.69 \\
\hline
\end{tabular}

This table shows the observed frequency in percentage of exceptions for the alternative VaR models under $1 \%, 5 \%, 10 \%$ and $25 \%$ signficance levels. An exception is defined by the indicator variable $I_{m t+1}$ for whether an exception occurrence is constructed as 1 if $R_{p t+1}<V a R_{m t}(\alpha)$, where $R_{p t+1}$ is the portfolio return on day $t+1$ and $\operatorname{VaR}_{m t}(\alpha)$ is the Value-at-Risk calculated on the previous business day with significance level $\alpha$ and using covariance specification $m$. 
Table 6: Unconditional Coverage Test Results

\begin{tabular}{|c|c|c|c|c|c|c|c|c|c|c|c|c|c|c|c|c|}
\hline & \multicolumn{4}{|c|}{ Normal distribution } & \multicolumn{4}{|c|}{ t-distribution } & \multicolumn{4}{|c|}{ Generalized t-distribution } & \multicolumn{4}{|c|}{ Nonparametric distribution } \\
\hline & $1 \%$ & $5 \%$ & $10 \%$ & $25 \%$ & $1 \%$ & $5 \%$ & $10 \%$ & $25 \%$ & $1 \%$ & $5 \%$ & $10 \%$ & $25 \%$ & $1 \%$ & $5 \%$ & $10 \%$ & $25 \%$ \\
\hline BEKK & 0.136 & 0.059 & 0.000 & 0.000 & 0.084 & 0.000 & 0.000 & 0.000 & 0.018 & 0.054 & 0.000 & 0.000 & 0.478 & 0.126 & 0.077 & 0.449 \\
\hline BEKK-L & 0.334 & 0.039 & 0.000 & 0.000 & 0.053 & .000 & 0.000 & .000 & .190 & 0.000 & 0.000 & 0.000 & .895 & 0.207 & 0.058 & 0.375 \\
\hline CCORR & 0.253 & 0.000 & 0.000 & 00 & 002 & 000 & 000 & 00 & 000 & 0.000 & 0.209 & 0.914 & 53 & 0.000 & 0.000 & 0.001 \\
\hline CCORR-L & 0.253 & 0.000 & 0.000 & 0.000 & 0.002 & 0.000 & 0.000 & 0.000 & 0.000 & 0.000 & 0.000 & 0.011 & 0.084 & 0.000 & 0.000 & 0.001 \\
\hline GDC & 0.670 & 0.000 & 0.000 & 0.000 & 000 & 000 & 0.000 & 0.000 & 000 & 0.004 & 0.000 & 0.000 & 0.053 & 0.000 & 0.000 & 0.023 \\
\hline GDC-L & & & & & & 000 & & & 00 & 003 & & & & & & 0.011 \\
\hline VECH & 0.431 & 0.019 & 0.000 & 0.000 & 0.018 & 0.000 & 0.000 & 0.000 & 0.190 & 0.000 & 0.000 & 0.000 & 0.268 & 0.019 & 0.077 & 0.424 \\
\hline VECH-L & 0.431 & 0.012 & 0.000 & & 0.018 & 000 & 0.000 & 0.000 & .190 & 0.000 & 0.000 & 0.000 & 90 & 0.012 & 0.043 & 0.375 \\
\hline E250 & .005 & 0.106 & 0.003 & 0.000 & 053 & 000 & 0.000 & 0.000 & .000 & 0.000 & 0.077 & 0.375 & 0.097 & 0.048 & 0.130 & 0.589 \\
\hline Eall & 0.067 & 0.012 & 0.000 & 0.000 & 0.005 & 000 & 0.000 & 0.000 & 000 & 0.000 & 0.000 & 0.000 & 0.129 & 0.000 & 0.001 & 0.147 \\
\hline EWMA & 000 & 0.966 & 0.037 & 0.007 & 0.031 & 000 & 0.000 & 0.000 & 0.000 & 0.000 & 0.000 & 0.072 & 0.129 & 0.088 & 0.232 & 0.038 \\
\hline E250 Port & 0.008 & 0.106 & 0.001 & 0.000 & 0.364 & 0.000 & 0.000 & 0.000 & 0.431 & 0.000 & 0.000 & 0.000 & 0.067 & 0.106 & 0.708 & 0.505 \\
\hline Eall Port & 0.188 & 0.001 & 0.000 & 0.000 & 0.009 & 0.000 & 0.000 & 0.000 & 0.000 & 0.000 & 0.000 & 0.002 & 0.190 & 0.001 & 0.000 & 0.003 \\
\hline EWMA Port & .005 & 0.831 & 0.058 & 0.008 & 0.190 & 0.001 & 0.000 & 0.000 & 0.000 & 0.009 & 0.380 & 0.402 & 0.365 & 0.318 & 0.380 & 0.427 \\
\hline GARCH Port & 0.543 & 0.003 & 0.000 & 0.000 & 0.018 & 0.000 & 0.000 & 0.000 & 0.084 & 0.000 & 0.000 & 0.000 & 0.478 & 0.003 & 0.007 & 0.104 \\
\hline
\end{tabular}

This table shows the p-value for the $L R_{u c}$ statistics for the alternative VaR models under $1 \%, 5 \%, 10 \%$ and $25 \%$ significance levels. The $L R_{u c}$ statistics are asymptotically distributed $\mathcal{X}^{2}(1)$. The cells in bold font indicate rejection of the null hypothesis of correct VaR estimates at the $5 \%$ significance level. 
Table 7: Conditional Coverage Test Results

\begin{tabular}{|c|c|c|c|c|c|c|c|c|c|c|c|c|c|c|c|c|}
\hline & \multicolumn{4}{|c|}{$\overline{\text { Normal distribution }}$} & \multicolumn{4}{|c|}{ t-distribution } & \multicolumn{4}{|c|}{ Generalized t-distribution } & \multicolumn{4}{|c|}{ " nonparametric distribution } \\
\hline & $1 \%$ & $5 \%$ & $10 \%$ & $25 \%$ & $1 \%$ & $5 \%$ & $10 \%$ & $25 \%$ & $1 \%$ & $5 \%$ & $10 \%$ & $25 \%$ & $1 \%$ & $5 \%$ & $10 \%$ & $25 \%$ \\
\hline BEKK & 0.203 & 0.082 & 0.000 & 0.000 & 0.196 & 0.000 & 0.000 & 0.000 & 0.054 & 0.000 & 0.000 & 0.000 & .625 & 0.183 & 0.074 & 0.692 \\
\hline BEKK-L & 0.416 & 0.081 & 0.000 & .000 & 0.135 & 000 & 0.000 & .000 & 0.358 & 0.000 & 0.000 & 0.000 & .753 & 0.300 & 0.051 & 0.609 \\
\hline CCORR & 0.337 & 0.000 & 0.000 & 000 & 009 & 000 & 0.000 & 000 & 000 & 0.000 & 142 & 0.992 & 135 & 0.000 & 0.000 & 0.003 \\
\hline CCORR-L & 0.337 & 0.000 & 0.000 & 0.000 & 0.009 & .000 & 0.000 & 0.000 & 0.000 & 0.000 & 0.000 & 0.040 & 0.196 & 0.000 & 0.000 & 0.004 \\
\hline GDC & 0.650 & 0.001 & 0.000 & 0.000 & 0.002 & 0.000 & 0.000 & 0.000 & 0.001 & 0.002 & 0.000 & 0.000 & 0.135 & 0.000 & 0.000 & 0.075 \\
\hline GDC-L & 0.650 & 0.002 & 0.000 & 0.000 & 0.009 & 0.000 & 0.000 & 0.000 & 0.002 & 0.002 & 0.000 & 0.000 & 0.196 & 0.000 & 0.000 & 0.040 \\
\hline VECH & 0.499 & 0.040 & 0.000 & 0.000 & 0.054 & 0.000 & 0.000 & 0.000 & 0.358 & 0.000 & 0.000 & 0.000 & 0.450 & 0.040 & 0.074 & 0.732 \\
\hline VECH-L & 0.499 & 0.023 & 0.000 & 0.000 & 0.054 & .000 & 0.000 & 0.000 & 0.358 & 0.000 & 0.000 & 0.000 & 0.358 & 0.023 & 0.048 & 0.681 \\
\hline E250 & 0.008 & 0.010 & 0.000 & 0.000 & 0.135 & 0.000 & 0.000 & 0.000 & 0.000 & 0.000 & 0.015 & 0.528 & 0.206 & 0.008 & 0.022 & 0.735 \\
\hline Eall & 0.109 & 0.000 & 0.000 & 0.000 & 0.017 & .000 & 0.000 & 0.000 & 0.000 & 0.000 & 0.000 & 0.000 & 0.272 & 0.000 & 0.001 & 0.338 \\
\hline EWMA & .001 & 0.384 & 0.053 & 0.028 & 0.088 & 000 & 0.000 & 0.000 & 0.000 & 0.000 & 0.000 & 0.201 & 0.272 & 0.125 & 0.279 & 0.110 \\
\hline E250 Port & 0.027 & 0.010 & 0.000 & 0.000 & 0.542 & 0.000 & 0.000 & 0.000 & 0.511 & 0.000 & 0.000 & 0.000 & 0.157 & 0.010 & 0.065 & 0.784 \\
\hline Eall Port & 0.265 & 0.000 & 0.000 & 0.000 & 0.031 & 0.000 & 0.000 & 0.000 & 0.000 & 0.000 & 0.000 & 0.005 & 0.359 & 0.000 & 0.000 & 0.012 \\
\hline EWMA Por & 0.018 & 0.421 & 0.091 & 0.032 & 0.358 & 0.002 & 0.000 & 0.000 & 0.000 & 0.010 & 0.389 & 0.688 & 0.542 & 0.186 & 0.389 & 0.706 \\
\hline GARCH Port & 0.579 & 0.005 & 0.000 & 0.000 & 0.054 & 0.000 & 0.000 & 0.000 & 0.196 & 0.000 & 0.000 & 0.000 & 0.625 & 0.005 & 0.006 & 0.266 \\
\hline
\end{tabular}

This table shows the p-value for the $L R_{c c}$ statistics for the alternative VaR models under $1 \%, 5 \%, 10 \%$ and $25 \%$ significance levels. The $L R_{c c}$ statistics are asymptotically distributed $\mathcal{X}^{2}(2)$. The cells in bold font indicate rejection of the null hypothesis of correct VaR estimates at the $5 \%$ significance level. 
Table 8: Dynamic Quantile Test Results

\begin{tabular}{|c|c|c|c|c|c|c|c|c|c|c|c|c|c|c|c|c|}
\hline & \multicolumn{4}{|c|}{ Normal distribution } & \multicolumn{4}{|c|}{ t-distribution } & \multicolumn{4}{|c|}{ Generalized t-distribution } & \multicolumn{4}{|c|}{ Nonparametric distribution } \\
\hline & $1 \%$ & $5 \%$ & $10 \%$ & $25 \%$ & $1 \%$ & $5 \%$ & $10 \%$ & $25 \%$ & $1 \%$ & $5 \%$ & $10 \%$ & $25 \%$ & $1 \%$ & $5 \%$ & $10 \%$ & $25 \%$ \\
\hline BEKK & 0.707 & 0.090 & 0.000 & 0.001 & 0.522 & 0.000 & 0.000 & 0.000 & 0.159 & 0.001 & 0.000 & 0.000 & 0.956 & 0.111 & 0.097 & 0.311 \\
\hline BEKK-L & 0.877 & 0.090 & 0.000 & 0.000 & 0.435 & 0.000 & 0.000 & 0.000 & 0.786 & 0.000 & 0.000 & 0.000 & 0.959 & 0.194 & 0.083 & 0.323 \\
\hline CCORR & 0.813 & 0.000 & 0.000 & 0.000 & 0.006 & 0.000 & 0.000 & 0.000 & 0.000 & 0.000 & 0.515 & 0.347 & 0.365 & 0.000 & 0.000 & 0.014 \\
\hline CCORR-L & 0.813 & 0.000 & 0.000 & 0.000 & 0.006 & 0.000 & 0.000 & 0.000 & 0.000 & 0.000 & 0.000 & 0.090 & 0.485 & 0.000 & 0.000 & 0.020 \\
\hline GDC & 0.962 & 0.001 & 0.000 & 0.000 & 0.000 & 0.000 & 0.000 & 0.000 & 0.000 & 0.033 & 0.000 & 0.000 & 0.425 & 0.000 & 0.002 & 0.171 \\
\hline GDC-L & 0.960 & 0.004 & 0.000 & & & 0.000 & 0.000 & 0.000 & 0.000 & 0.038 & 0.000 & 0.000 & 0.573 & 0.000 & 0.001 & 0.097 \\
\hline VECH & 0.938 & 0.067 & 0.000 & 0.000 & 0.154 & 0.000 & 0.000 & 0.000 & 0.793 & 0.000 & 0.000 & 0.000 & 0.841 & 0.067 & 0.201 & 0.498 \\
\hline VECH-L & 0.938 & 0.068 & 0.000 & 0.000 & 0.153 & 0.000 & 0.000 & 0.000 & 0.792 & 0.000 & 0.000 & 0.000 & 0.792 & 0.068 & 0.144 & 0.468 \\
\hline E250 & 0.017 & 0.000 & 0.000 & 0.000 & 0.145 & 0.000 & 0.000 & 0.000 & 0.000 & 0.000 & 0.000 & 0.004 & 0.438 & 0.000 & 0.000 & 0.014 \\
\hline Eall & 0.213 & 0.000 & 0.000 & 0.000 & 0.042 & 0.000 & 0.000 & 0.000 & 0.000 & 0.000 & 0.000 & 0.000 & 0.383 & 0.000 & 0.000 & 0.333 \\
\hline EWMA & 0.043 & 0.104 & 0.018 & 0.021 & 0.173 & 0.000 & 0.000 & 0.000 & 0.000 & 0.000 & 0.000 & 0.132 & 0.516 & 0.013 & 0.035 & 0.024 \\
\hline E250 Port & 0.035 & 0.000 & 0.000 & 0.000 & 0.832 & 0.000 & 0.000 & 0.000 & 0.314 & 0.000 & 0.000 & 0.000 & 0.367 & 0.000 & 0.001 & 0.042 \\
\hline Eall Port & 0.140 & 0.000 & 0.000 & 0.000 & 0.066 & 0.000 & 0.000 & 0.000 & 0.000 & 0.000 & 0.000 & 0.050 & 0.486 & 0.000 & 0.000 & 0.059 \\
\hline EWMA Port & 0.053 & 0.120 & 0.023 & 0.023 & 0.528 & 0.000 & 0.000 & 0.000 & 0.000 & 0.000 & 0.044 & 0.168 & 0.772 & 0.019 & 0.044 & 0.135 \\
\hline GARCH Port & 0.950 & 0.015 & 0.000 & 0.000 & 0.162 & 0.000 & 0.000 & 0.000 & 0.572 & 0.000 & 0.000 & 0.000 & 0.939 & 0.015 & 0.039 & 0.225 \\
\hline
\end{tabular}

This table shows the p-value for the $D Q$ statistic for the alternative VaR models under $1 \%, 5 \%, 10 \%$ and $25 \%$ signficance levels. The $D Q$ statistics are asymptotically distributed $\mathcal{X}^{2}(7)$. The cells in bold font indicate rejection of the null hypothesis of correct VaR estimates at the $5 \%$ significance level. 
Table 9: Distribution Forecast Test Results

\begin{tabular}{lrrrr}
\hline \hline & $\begin{array}{c}\text { Normal } \\
\text { distribution }\end{array}$ & t-distribution & $\begin{array}{c}\text { Generalized } \\
\text { t-distribution }\end{array}$ & $\begin{array}{c}\text { Nonparametric } \\
\text { distribution }\end{array}$ \\
\hline BEKK & 0.433 & $\mathbf{0 . 0 0 0}$ & 0.520 & 0.265 \\
BEKK-L & 0.329 & $\mathbf{0 . 0 0 0}$ & 0.475 & 0.381 \\
CCORR & $\mathbf{0 . 0 0 0}$ & $\mathbf{0 . 0 0 0}$ & $\mathbf{0 . 0 0 0}$ & $\mathbf{0 . 0 0 0}$ \\
CCORR-L & $\mathbf{0 . 0 0 0}$ & $\mathbf{0 . 0 0 0}$ & $\mathbf{0 . 0 0 0}$ & $\mathbf{0 . 0 0 0}$ \\
GDC & $\mathbf{0 . 0 0 1}$ & $\mathbf{0 . 0 0 0}$ & $\mathbf{0 . 0 1 6}$ & $\mathbf{0 . 0 0 0}$ \\
GDC-L & $\mathbf{0 . 0 1 1}$ & $\mathbf{0 . 0 0 0}$ & $\mathbf{0 . 0 4 1}$ & $\mathbf{0 . 0 0 0}$ \\
VECH & 0.204 & $\mathbf{0 . 0 0 0}$ & 0.510 & 0.090 \\
VECH-L & 0.162 & $\mathbf{0 . 0 0 0}$ & 0.507 & 0.119 \\
E250 & 0.298 & $\mathbf{0 . 0 0 0}$ & 0.142 & 0.536 \\
Eall & 0.160 & $\mathbf{0 . 0 0 0}$ & $\mathbf{0 . 0 0 0}$ & $\mathbf{0 . 0 0 0}$ \\
EWMA & $\mathbf{0 . 0 0 0}$ & $\mathbf{0 . 0 0 0}$ & 0.231 & 0.135 \\
E250 Port & 0.360 & $\mathbf{0 . 0 0 0}$ & 0.570 & $\mathbf{0 . 0 1 2}$ \\
Eall Port & $\mathbf{0 . 0 0 1}$ & $\mathbf{0 . 0 0 0}$ & $\mathbf{0 . 0 0 2}$ & $\mathbf{0 . 0 0 0}$ \\
EWMA Port & $\mathbf{0 . 0 0 0}$ & $\mathbf{0 . 0 0 0}$ & 0.188 & 0.804 \\
GARCH Port & $\mathbf{0 . 0 0 3}$ & $\mathbf{0 . 0 0 0}$ & $\mathbf{0 . 0 3 5}$ & $\mathbf{0 . 0 0 4}$ \\
\hline \hline
\end{tabular}

This table shows the p-value for the $L R_{\text {dist }}$ statistics for the alternative VaR models under $1 \%, 5 \%, 10 \%$ and $25 \%$ quantiles. The $L R_{\text {dist }}$ statistic is asymptotically distributed $\mathcal{X}^{2}(3)$. The cells in bold font indicate rejection of the null hypothesis of correct VaR estimates at the $5 \%$ significance level. 
Table 10: Mean Exception

\begin{tabular}{|c|c|c|c|c|c|c|c|c|c|c|c|c|c|c|c|c|}
\hline & \multicolumn{4}{|c|}{ Normal distribution } & \multicolumn{4}{|c|}{ t-distribution } & \multicolumn{4}{|c|}{ Generalized t-distribution } & \multicolumn{4}{|c|}{ Nonparametric distribution } \\
\hline & $1 \%$ & $5 \%$ & $10 \%$ & $25 \%$ & $1 \%$ & $5 \%$ & $10 \%$ & $25 \%$ & $1 \%$ & $5 \%$ & $10 \%$ & $25 \%$ & $1 \%$ & $5 \%$ & $10 \%$ & $25 \%$ \\
\hline BEKK & -0.579 & -0.633 & -0.646 & -0.686 & -0.483 & -0.655 & -0.673 & -0.685 & -0.458 & -0.660 & -0.675 & -0.724 & -0.563 & -0.640 & -0.667 & -0.709 \\
\hline BEKK-L & -0.602 & -0.630 & -0.641 & -0.688 & -0.483 & -0.648 & -0.661 & -0.682 & -0.567 & -0.658 & -0.677 & -0.727 & -0.643 & -0.631 & -0.669 & -0.706 \\
\hline CCORR & -0.551 & -0.671 & -0.667 & -0.681 & -0.524 & -0.691 & -0.663 & -0.678 & -0.598 & -0.658 & -0.651 & -0.696 & -0.537 & -0.676 & -0.642 & -0.698 \\
\hline CCORR-L & -0.551 & -0.671 & -0.667 & -0.681 & -0.524 & -0.690 & -0.663 & -0.679 & -0.477 & -0.674 & -0.648 & -0.697 & -0.527 & -0.677 & -0.644 & -0.696 \\
\hline GDC & -0.603 & -0.651 & -0.640 & -0.681 & -0.612 & -0.638 & -0.666 & -0.673 & -0.619 & -0.668 & -0.660 & -0.720 & -0.528 & -0.648 & -0.645 & -0.693 \\
\hline GDC-L & -0.620 & -0.650 & -0.646 & & & & & & & -0.671 & -0.660 & & -0.526 & -0.661 & -0.639 & -0.695 \\
\hline VECH & -0.634 & -0.623 & -0.648 & -0.687 & -0.556 & -0.615 & -0.646 & -0.678 & -0.573 & -0.649 & -0.671 & -0.718 & -0.567 & -0.622 & -0.655 & -0.696 \\
\hline VECH-L & -0.627 & -0.628 & -0.646 & -0.686 & -0.549 & -0.616 & -0.642 & -0.679 & -0.566 & -0.648 & -0.671 & -0.722 & -0.584 & -0.628 & -0.659 & -0.695 \\
\hline E250 & -0.614 & -0.698 & -0.668 & -0.724 & -0.531 & -0.681 & -0.654 & -0.718 & -0.593 & -0.689 & -0.666 & -0.717 & -0.533 & -0.676 & -0.672 & -0.714 \\
\hline Eall & -0.650 & -0.700 & -0.675 & -0.713 & -0.569 & -0.718 & -0.713 & -0.703 & -0.761 & -0.707 & -0.708 & -0.715 & -0.684 & -0.719 & -0.681 & -0.736 \\
\hline EWMA & -0.510 & -0.601 & -0.638 & -0.677 & -0.498 & -0.586 & -0.618 & -0.689 & -0.366 & -0.552 & -0.614 & -0.670 & -0.514 & -0.598 & -0.643 & -0.701 \\
\hline E250 Port & -0.622 & -0.694 & -0.674 & -0.723 & -0.513 & -0.697 & -0.655 & -0.719 & -0.499 & -0.668 & -0.691 & -0.742 & -0.556 & -0.708 & -0.678 & -0.716 \\
\hline Eall Port & -0.621 & -0.703 & -0.695 & -0.711 & -0.593 & -0.713 & -0.726 & -0.700 & -0.759 & -0.684 & -0.680 & -0.735 & -0.659 & -0.704 & -0.687 & -0.737 \\
\hline EWMA Port & -0.556 & -0.590 & -0.630 & -0.676 & -0.470 & -0.583 & -0.607 & -0.687 & -0.374 & -0.587 & -0.638 & -0.691 & -0.558 & -0.608 & -0.645 & -0.691 \\
\hline GARCH Port & -0.610 & -0.640 & -0.652 & -0.689 & -0.545 & -0.663 & -0.668 & -0.688 & -0.573 & -0.649 & -0.679 & -0.731 & -0.554 & -0.633 & -0.660 & -0.704 \\
\hline
\end{tabular}

This table shows mean exception in excess of the VaR conditional on the portfolio return being lower than the VaR estimate in each day. Mean exception is shown in percentage for the alternative VaR models under the $1 \%, 5 \%, 10 \%$ and $25 \%$ significance levels. 
Table 11: Normal Transform Test Results

\begin{tabular}{|c|c|c|c|c|c|c|c|c|c|c|c|c|c|c|c|c|}
\hline & \multicolumn{4}{|c|}{ Normal distribution } & \multicolumn{4}{|c|}{ t-distribution } & \multicolumn{4}{|c|}{ Generalized t-distribution } & \multicolumn{4}{|c|}{ Nonparametric distribution } \\
\hline & $1 \%$ & $5 \%$ & $10 \%$ & $25 \%$ & $1 \%$ & $5 \%$ & $10 \%$ & $25 \%$ & $1 \%$ & $5 \%$ & $10 \%$ & $25 \%$ & $1 \%$ & $5 \%$ & $10 \%$ & $25 \%$ \\
\hline BEKK & 0.208 & 0.007 & 0.000 & 0.000 & 0.000 & 0.000 & 0.000 & 0.000 & 0.635 & 0.789 & 0.072 & 0.000 & 0.035 & 0.000 & 0.000 & 0.001 \\
\hline BEKK-L & 0.097 & 0.000 & 0.000 & 0.000 & 0.000 & 0.000 & 0.000 & 0.000 & 0.032 & 0.000 & 0.000 & 0.000 & 0.120 & 0.006 & 0.007 & 0.828 \\
\hline CCORR & 0.000 & 0.000 & 0.000 & 0.000 & 0.000 & 0.000 & 0.000 & 0.000 & 0.000 & 0.000 & 0.000 & 0.000 & 0.000 & 0.000 & 0.000 & 0.000 \\
\hline CCORR-L & 0.000 & 0.000 & 0.000 & 0.000 & 0.000 & 0.000 & 0.000 & 0.000 & 0.000 & 0.000 & 0.000 & 0.000 & 0.000 & 0.000 & 0.000 & 0.000 \\
\hline GDC & 0.002 & 0.000 & 0.000 & 0.000 & 0.000 & 0.000 & 0.000 & 0.000 & 0.002 & 0.000 & 0.000 & 0.142 & 0.000 & 0.000 & 0.000 & 0.000 \\
\hline GDC-L & 0.005 & 0.000 & 0.000 & 0.000 & 0.000 & 0.000 & 0.000 & 0.000 & 0.017 & 0.000 & 0.000 & 0.000 & 0.000 & 0.000 & 0.000 & 0.000 \\
\hline VECH & 0.076 & 0.000 & 0.000 & 0.000 & 0.000 & 0.000 & 0.000 & 0.000 & 0.094 & 0.000 & 0.000 & 0.000 & 0.023 & 0.000 & 0.000 & 0.017 \\
\hline VECH-L & 0.053 & 0.000 & 0.000 & 0.000 & 0.000 & 0.000 & 0.000 & 0.000 & 0.132 & 0.000 & 0.000 & 0.000 & 0.031 & 0.000 & 0.000 & 0.035 \\
\hline E250 & 0.078 & 0.001 & 0.000 & 0.000 & 0.000 & 0.000 & 0.000 & 0.000 & 0.015 & 0.000 & 0.000 & 0.000 & 0.320 & 0.227 & 0.386 & 0.001 \\
\hline Eall & 0.054 & 0.000 & 0.000 & 0.000 & 0.000 & 0.000 & 0.000 & 0.000 & 0.000 & 0.000 & 0.000 & 0.023 & 0.000 & 0.000 & 0.000 & 0.000 \\
\hline EWMA & 0.000 & 0.000 & 0.000 & 0.000 & 0.000 & 0.000 & 0.000 & 0.000 & 0.050 & 0.000 & 0.000 & 0.000 & 0.015 & 0.000 & 0.000 & 0.000 \\
\hline E250 Port & 0.100 & 0.001 & 0.000 & 0.000 & 0.000 & 0.000 & 0.000 & 0.000 & 0.012 & 0.000 & 0.000 & 0.000 & 0.000 & 0.000 & 0.000 & 0.000 \\
\hline Eall Port & 0.002 & 0.000 & 0.000 & 0.000 & 0.000 & 0.000 & 0.000 & 0.000 & 0.000 & 0.000 & 0.000 & 0.000 & 0.000 & 0.000 & 0.000 & 0.000 \\
\hline EWMA Port & 0.003 & 0.000 & 0.000 & 0.000 & 0.000 & 0.000 & 0.000 & 0.000 & 0.002 & 0.000 & 0.000 & 0.000 & 0.785 & 0.479 & 0.350 & 0.243 \\
\hline GARCH Port & 0.004 & 0.000 & 0.000 & 0.000 & 0.000 & 0.000 & 0.000 & 0.000 & 0.001 & 0.000 & 0.000 & 0.000 & 0.001 & 0.000 & 0.000 & 0.000 \\
\hline
\end{tabular}

This table shows the p-value for the $L R_{\text {mag }}$ test statistic for the alternative VaR models under $1 \%, 5 \%, 10 \%$ and $25 \%$ significance levels. The $L R_{m a g}$ statistic is asymptotically distributed $\mathcal{X}^{2}(2)$. The cells in bold font indicate rejection of the null hypothesis of correct VaR estimates at the $5 \%$ significance level. 
Table 12: Capital Charge Relative to Benchmark Model - EWMA-Normal

\begin{tabular}{lrrrrrrrr}
\hline \hline & \multicolumn{2}{c}{ Normal distrib. } & \multicolumn{2}{c}{ t-distribution } & \multicolumn{3}{c}{ Generalized t-distrib. } & \multicolumn{2}{c}{ Nonparametric distrib. } \\
\cline { 2 - 8 } & \% trading & p-value & \% trading & p-value & \% trading & p-value & \% trading & p-value \\
\hline BEKK & 57.11 & 0.539 & 87.14 & $\mathbf{0 . 0 0 0}$ & 89.66 & $\mathbf{0 . 0 0 0}$ & 70.43 & $\mathbf{0 . 0 1 0}$ \\
BEKK-L & 55.40 & 0.696 & 87.33 & $\mathbf{0 . 0 0 0}$ & 78.14 & $\mathbf{0 . 0 0 0}$ & 58.34 & 0.246 \\
CCORR & 67.45 & $\mathbf{0 . 0 1 0}$ & 92.52 & $\mathbf{0 . 0 0 0}$ & 98.89 & $\mathbf{0 . 0 0 0}$ & 87.29 & $\mathbf{0 . 0 0 0}$ \\
CCORR-L & 67.45 & $\mathbf{0 . 0 1 0}$ & 92.56 & $\mathbf{0 . 0 0 0}$ & 100.00 & $\mathbf{0 . 0 0 0}$ & 87.03 & $\mathbf{0 . 0 0 0}$ \\
GDC & 60.70 & 0.072 & 93.78 & $\mathbf{0 . 0 0 0}$ & 93.82 & $\mathbf{0 . 0 0 0}$ & 83.29 & $\mathbf{0 . 0 0 0}$ \\
GDC-L & 60.02 & 0.140 & 92.98 & $\mathbf{0 . 0 0 0}$ & 93.86 & $\mathbf{0 . 0 0 0}$ & 81.34 & $\mathbf{0 . 0 0 0}$ \\
VECH & 61.92 & 0.221 & 90.11 & $\mathbf{0 . 0 0 0}$ & 80.20 & $\mathbf{0 . 0 0 0}$ & 77.34 & $\mathbf{0 . 0 0 0}$ \\
VECH-L & 63.37 & 0.168 & 90.35 & $\mathbf{0 . 0 0 0}$ & 81.19 & $\mathbf{0 . 0 0 0}$ & 78.79 & $\mathbf{0 . 0 0 0}$ \\
E250 & 58.64 & 0.256 & 79.55 & $\mathbf{0 . 0 0 0}$ & 97.48 & $\mathbf{0 . 0 0 0}$ & 67.65 & $\mathbf{0 . 0 2 8}$ \\
Eall & 65.01 & 0.211 & 84.74 & $\mathbf{0 . 0 0 0}$ & 97.60 & $\mathbf{0 . 0 0 0}$ & 71.96 & $\mathbf{0 . 0 1 0}$ \\
EWMA & - & - & 99.35 & $\mathbf{0 . 0 0 0}$ & 100.00 & $\mathbf{0 . 0 0 0}$ & 92.56 & $\mathbf{0 . 0 0 0}$ \\
E250 Port & 57.76 & 0.240 & 77.45 & $\mathbf{0 . 0 0 0}$ & 70.89 & $\mathbf{0 . 0 0 0}$ & 65.82 & 0.050 \\
Eall Port & 68.45 & 0.123 & 83.94 & $\mathbf{0 . 0 0 0}$ & 94.35 & $\mathbf{0 . 0 0 0}$ & 71.92 & $\mathbf{0 . 0 1 2}$ \\
EWMA Port & 36.28 & 0.996 & 93.02 & $\mathbf{0 . 0 0 0}$ & 100.00 & $\mathbf{0 . 0 0 0}$ & 82.07 & $\mathbf{0 . 0 0 0}$ \\
GARCH Port & 61.92 & 0.165 & 86.91 & $\mathbf{0 . 0 0 0}$ & 80.24 & $\mathbf{0 . 0 0 0}$ & 77.30 & $\mathbf{0 . 0 0 0}$ \\
\hline \hline
\end{tabular}

This table shows the percentage of trading days for which the benchmark VaR model (EWMA-Normal) has lower capital charge than the one given by the each model. The cells in bold font indicate rejection of the null hypothesis of equal capital charges at the $5 \%$ significance level using the Diebold-Mariano test statistic. 
Table 13: Capital Charge Relative to Benchmark Model - EWMA Portfolio-Normal

\begin{tabular}{|c|c|c|c|c|c|c|c|c|}
\hline & \multicolumn{2}{|c|}{ Normal distrib. } & \multicolumn{2}{|c|}{ t-distrib. } & \multicolumn{2}{|c|}{ Generalized t-distrib. } & \multicolumn{2}{|c|}{ Nonparametric distrib. } \\
\hline & $\%$ trading & p-value & $\%$ trading & p-value & $\%$ trading & p-value & $\%$ trading & $\overline{p \text {-value }}$ \\
\hline BEKK & 66.34 & 0.039 & 96.49 & 0.000 & $\overline{96.22}$ & 0.000 & 76.50 & 0.000 \\
\hline BEKK-L & 63.56 & 0.087 & 94.20 & 0.000 & 86.99 & 0.000 & 69.29 & 0.003 \\
\hline CCORR & 75.93 & 0.000 & 95.23 & 0.000 & 100.00 & 0.000 & 95.77 & 0.000 \\
\hline CCORR-L & 75.96 & 0.000 & 95.23 & 0.000 & 100.00 & 0.000 & 95.42 & 0.000 \\
\hline GDC & 73.03 & 0.000 & 96.11 & 0.000 & 96.30 & 0.000 & 90.92 & 0.000 \\
\hline GDC-L & 71.96 & 0.001 & 96.07 & 0.000 & 96.22 & 0.000 & 90.23 & 0.000 \\
\hline VECH & 71.30 & 0.001 & 95.80 & 0.000 & 90.42 & 0.000 & 87.52 & 0.000 \\
\hline VECH-L & 72.15 & 0.001 & 95.84 & 0.000 & 91.11 & 0.000 & 88.33 & 0.000 \\
\hline E250 & 63.41 & 0.074 & 82.49 & 0.000 & 98.55 & 0.000 & 71.65 & 0.003 \\
\hline Eall & 68.49 & 0.054 & 88.36 & 0.000 & 99.73 & 0.000 & 75.43 & 0.001 \\
\hline EWMA & 63.72 & 0.004 & 100.00 & 0.000 & 100.00 & 0.000 & 99.85 & 0.000 \\
\hline E250 Port & 62.99 & 0.066 & 80.69 & 0.000 & 74.21 & 0.000 & 70.16 & 0.007 \\
\hline Eall Port & 71.69 & 0.025 & 86.88 & 0.000 & 96.80 & 0.000 & 75.39 & 0.001 \\
\hline EWMA Port & - & - & 99.85 & 0.000 & 100.00 & 0.000 & 93.82 & 0.000 \\
\hline GARCH Port & 70.89 & 0.003 & 92.60 & 0.000 & 86.53 & 0.000 & 84.59 & 0.000 \\
\hline
\end{tabular}

This table shows the percentage of trading days for which the benchmark VaR model (EWMAPortfolioNormal) has lower capital charge than the one given by the each model. The cells in bold font indicate rejection of the null hypothesis of equal capital charges at the $5 \%$ significance level using the Diebold-Mariano test statistic. 